ARTICLE

DOI: $10.1038 / \mathrm{s} 41467-018-05605-7$

\title{
AIP1 and cofilin ensure a resistance to tissue tension and promote directional cell rearrangement
}

\author{
Keisuke Ikawa ${ }^{1} \&$ Kaoru Sugimura (D) ${ }^{1,2}$
}

In order to understand how tissue mechanics shapes animal body, it is critical to clarify how cells respond to and resist tissue stress when undergoing morphogenetic processes, such as cell rearrangement. Here, we address the question in the Drosophila wing epithelium, where anisotropic tissue tension orients cell rearrangements. We found that anisotropic tissue tension localizes actin interacting protein 1 (AIP1), a cofactor of cofilin, on the remodeling junction via cooperative binding of cofilin to F-actin. AIP1 and cofilin promote actin turnover and locally regulate the Canoe-mediated linkage between actomyosin and the junction. This mechanism is essential for cells to resist the mechanical load imposed on the remodeling junction perpendicular to the direction of tissue stretching. Thus, the present study delineates how AIP1 and cofilin achieve an optimal balance between resistance to tissue tension and morphogenesis.

\footnotetext{
${ }^{1}$ Institute for Integrated Cell-Material Sciences (WPI-iCeMS), Kyoto University, Kyoto 606-8501, Japan. ${ }^{2}$ JST PRESTO, Tokyo 102-0075, Japan.

Correspondence and requests for materials should be addressed to K.S. (email: ksugimura@icems.kyoto-u.ac.jp)
} 
$\mathrm{T}$ he global patterns of forces in a tissue (e.g., tissue tension/ compression) control many aspects of development including cell proliferation, cell rearrangement, and cell polarity ${ }^{1-10}$. Such control relies on the ability of cells to sense the distribution of forces and tune morphogenetic signaling pathways in response to the mechanical inputs. Moreover, cells must resist or release tension/compression when deforming, proliferating, and moving during development ${ }^{2,11-13}$. While an understanding of molecular mechanisms for stress generation has evolved in the past decade, much less is known on how cells respond to and resist such stresses at the molecular level during morphogenesis.

The actin cytoskeleton is capable of sensing and resisting applied forces both at the network and filament levels ${ }^{14,15}$. For example, mechanical strain on the actin network alters the structure of filamin A, which crosslinks the orthogonal filaments, thus inhibiting the binding between filamin A and a downstream signaling molecule ${ }^{16}$. Single actin filaments decrease their helical pitch when mechanically relaxed, and such structural changes are amplified through positive feedback between F-actin twisting and cofilin binding ${ }^{15,17-19}$. The actin network increases its elasticity or reorients the stress direction to resist applied forces by changing filament dynamics and/or network architecture ${ }^{14,20,21}$. Whether and how these force-responsive properties of the actin cytoskeleton and actin-binding proteins (ABPs) are involved in the development of multi-cellular tissue is largely unknown.

During morphogenesis, cells change their relative positions along the tissue axis by remodeling cell contact surfaces. This process, called directional cell rearrangement, shapes a tissue and develops its multi-cellular pattern ${ }^{22-25}$. The Drosophila pupal wing epithelium provides an excellent model system to study the mechanism through which tissue tension controls directional cell rearrangement. Starting $\sim 15 \mathrm{~h}$ after puparium formation (h APF), forces generated in the hinge stretch the wing along the proximaldistal (PD) axis (Supplementary Figure 1a-d) ${ }^{6}$. The resulting anisotropic tissue tension acts as a mechanical cue to specify the axis of cell rearrangement ${ }^{6-8,26}$. Wing cells relocalize myosin-II (myo-II) at the adherens junction (AJ) that runs along the $\mathrm{PD}$ axis (PD junction) to resist tissue tension, and the balance between extrinsic stretching force and intrinsic cell junction tension favors PD cell rearrangement, thereby accelerating relaxation into a hexagonal cell pattern (hereafter called hexagonal cell packing; Supplementary Figure 1c, d) ${ }^{7}$. This relaxation may be primarily driven through interface mechanics, consistent with the observation of shear-induced reconnection of interfaces and hexagonal lattice formation in foam, non-biological soft matter ${ }^{27,28}$. However, in biological tissues such as the wing epithelium, interface mechanics must be orchestrated with molecular regulators of cytoskeleton and cell adhesion (e.g., force-responsive $\mathrm{ABPs}$ ) responsible for responding to and resisting tissue tension. Answering the question in the wing should provide a general mechanism of epithelial development, as all cell rearrangements are associated with sensation and resistance to forces from the surrounding cells.

Here, we show that actin regulation mediated through actin interacting protein 1 (AIP1) and cofilin is responsible for supporting tissue tension-driven cell rearrangement and hexagonal cell packing in the Drosophila pupal wing. AIP1 is evolutionarily conserved from yeast to humans. In vitro studies have shown that AIP1 binds cofilin and F-actin and promotes F-actin severing via cofilin $^{29-32}$. In vivo, AIP1 and cofilin control F-actin disassembly and remodeling during development ${ }^{33-38}$. We show that AIP1 is localized on the remodeling anterior-posterior (AP) junctions of wing cells, and tissue stretch is necessary for the biased distribution of AIP1. Inhibition of actin turnover by AIP1 or cofilin loss-of-function (l-o-f) results in the detachment of myo-II from the AP junctions, which hampers the stabilization of newly formed PD junctions. Interestingly, the disorder of junctional actomyosin is rescued by releasing tissue tension. Together, our data illustrate that actin turnover ensures a resistance to anisotropic tissue tension and promotes directional cell rearrangement by reinforcing the structural stability of remodeling junctions. This proposed mechanism is likely to be relevant to development of other epithelial tissues in which tissue tension coordinates with morphogenetic signaling pathways in individual cells.

\section{Results}

AIP1 is localized on remodeling AP junctions. To investigate the molecular mechanisms through which cells respond to and resist tissue tension during directional cell rearrangements in the Drosophila wing, we screened candidate ABPs (Supplementary Methods). First, we examined the subcellular distribution of 19 $\mathrm{ABPs}$ at $24 \mathrm{~h} \mathrm{APF}$. ABPs demonstrating interesting localization patterns were assayed for l-o-f phenotypes. Since the polygonal distribution of cells is much easier to measure than the dynamics of cell rearrangement, we used the fraction of hexagonal cells at $32 \mathrm{~h} \mathrm{APF}$, when the hexagonal cell packing process involving directional cell rearrangement is nearly complete, as a proxy for cell rearrangement defects. As described below, screening identified AIP1, which promotes F-actin severing via cofilin ${ }^{29-32}$, as a potential key regulator of cell rearrangement in the wing.

During the screening process, we detected a strong signal for GFP-tagged endogenous AIP1 along a subset of AP junctions at $24 \mathrm{~h} \mathrm{APF} \mathrm{(Fig.} \mathrm{1a,} \mathrm{b,} \mathrm{Supplementary} \mathrm{Figure} \mathrm{1e;} \mathrm{see} \mathrm{Supplementary}$ Note 1 and Supplementary Figure 2a, c for characterization of flare (flr) (Drosophila aip1 gene) protein trap line $\left.e^{36,39}\right)$. The directional bias in the AIP1 distribution became weaker at $28 \mathrm{~h}$ APF, when tissue tension is thought to contribute less to directional cell rearrangement (Supplementary Figure 1f, g) ${ }^{7}$. Time-lapse analysis confirmed that AIP1 accumulated on AP junctions during junctional remodeling, and AIP1 gradually disappeared from newly formed PD junctions (Fig. 1e,f; Supplementary Movie 1). In contrast, AIP1-GFP signal intensity remains low at stable AP junctions (Fig. 1g,h; Supplementary Movie 2), indicating that AIP1 specifically localizes to remodeling AP junctions. Fluorescence recovery after photobleaching (FRAP) of utrABD-GFP showed a smaller fraction of stable F-actin at the AP junctions than at the PD junction, which is in agreement with the AIP1 subcellular localization (Fig. 1i-l; Welch's $t$-test, $P<$ 0.01 ). Next, we examined the localization of AIP1 and its cofactor cofilin along the apico-basal axis. We confirmed that AIP1 is enriched at the AJ plane, whereas cofilin is diffusely distributed, as has been reported in the eye $\operatorname{disc}^{36}$ (Fig. 1c, d and Supplementary Figure 3f-h; see Supplementary Note 1 and Supplementary Figure 2b, c for characterization of twinstar (tsr) (Drosophila cofilin gene) protein trap line ${ }^{40}$ ). In the flr RNAi wing, hexagonal cell packing was disrupted, consistent with a defect in directional cell rearrangement (Fig. 2a, b, d; Welch's $t$ test, $P<0.001)$. These results prompted us to further investigate a role for AIP1 in tissue tension-driven cell rearrangement.

Tissue tension-dependent localization of AIP1. To characterize the mechanism by which tissue tension acts on AIP1, we examined whether tissue tension is required to bias the subcellular distribution of AIP1. To this end, we relaxed the tissue stretch by detaching the wing from the hinge $e^{6,7}$. AIP1 was more evenly distributed along junctions in the mechanically relaxed wing (Fig. 3a-d). WT wing cells accumulate F-actin along the PD rather than AP junctions, and such F-actin localization becomes non-polarized by relaxing the tissue stretch (Fig. 3e, f). Therefore, our data argue against the possibility that the loss of accumulation of AIP1 along the AP junctions in Fig. 3c, $d$ is simply a result of 
a
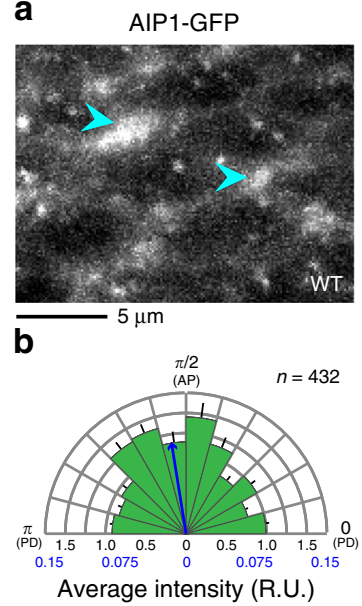

$R_{s}$

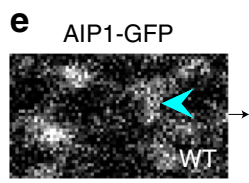

$24: 28$

AIP1-GFP
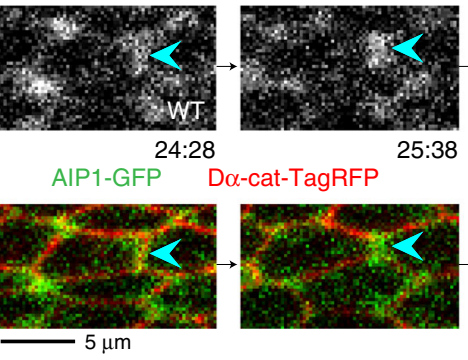

Do-cat-TagRFP

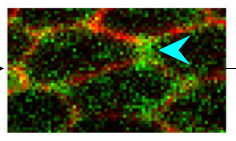

f

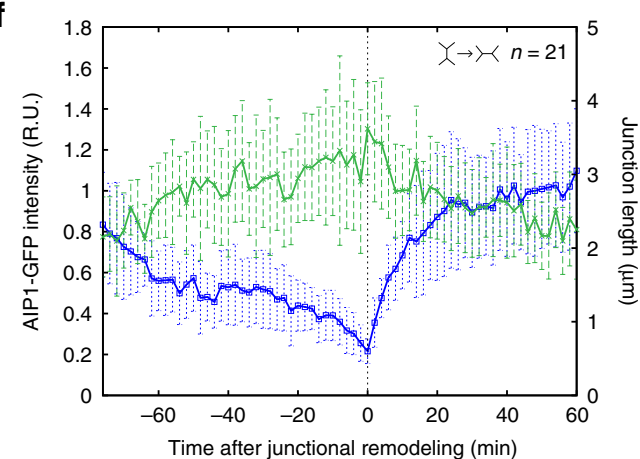

i

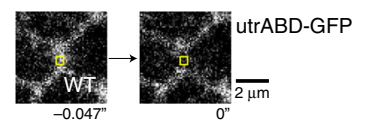

j
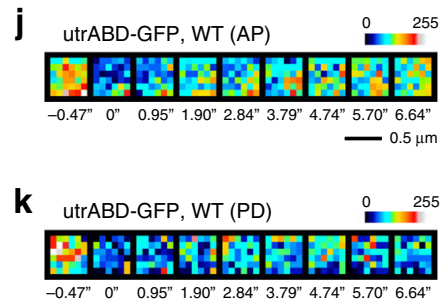

25:38

D $\alpha$-cat-TagRFP
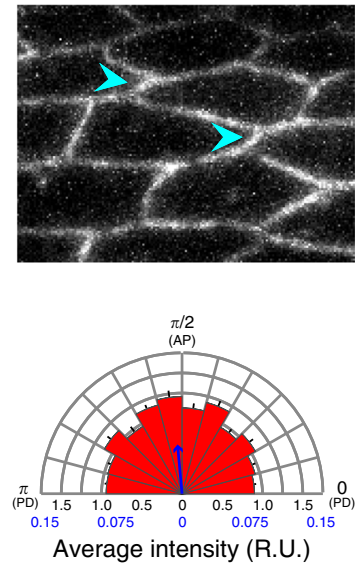

$R_{s}$

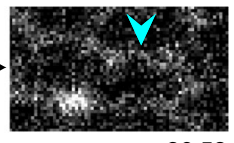

26:58

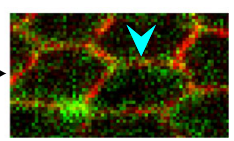

\section{(1)}
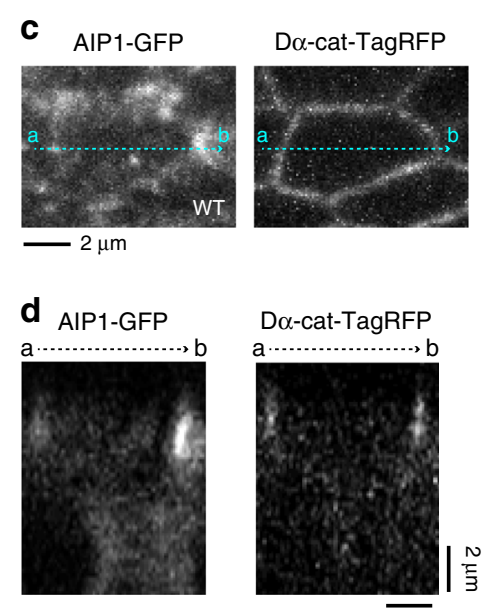

\section{g AIP1-GFP}
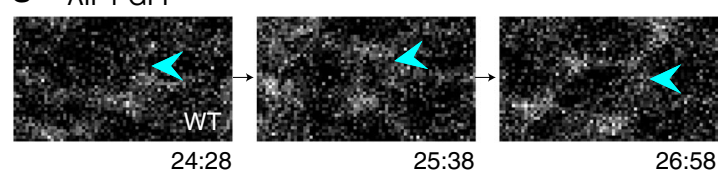

AIP1-GFP D $\alpha$-cat-TagRFP
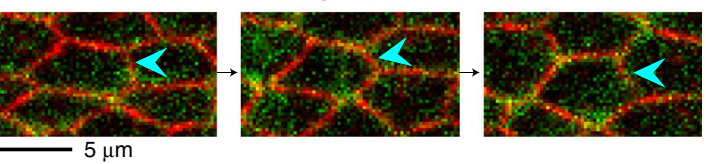

h

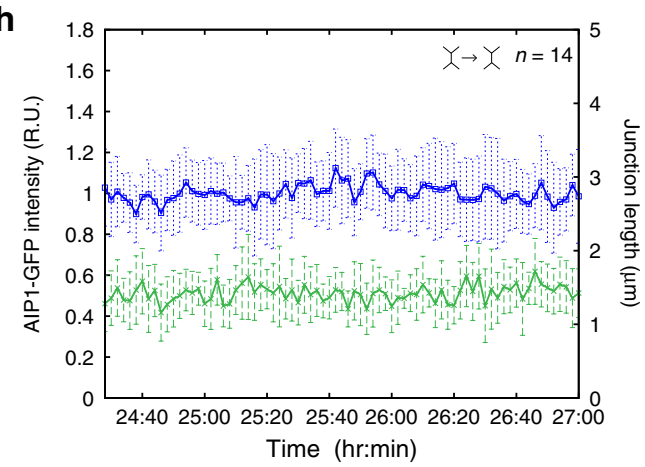

the change in F-actin localization. For comparison, the same experiment was performed with another ABP, Enabled (Ena), which promotes the elongation of actin filaments ${ }^{41}$. As shown in Fig. 3g, h, Ena retained its localization at junctions and vertices in the absence of an extrinsic stretching force. Thus, tissue tension- dependent subcellular localization is specific to a subset of ABPs, including AIP1 and myo-II 7 ,42.

In addition to mechanical anisotropy, the wing develops a planar cell polarity (PCP) along the in-plane axis of the epithelium $^{43}$, which may bias AIP1 localization. However, AIP1 
Fig. 1 AIP1 localizes to remodeling AP junctions in the Drosophila pupal wing. a AIP1-GFP (left) and an AJ marker, D $\alpha$-cat-TagRFP (right) in the WT wing at $24 \mathrm{~h}$ APF. Blue arrowheads indicate AP junctions. b Directional bias of AIP1-GFP signal intensity (left) and D $\alpha$-cat-TagRFP signal intensity (right) along the junctions. Each junction is divided into twelve bins according to its angle relative to the PD axis, and the average signal intensity in each bin is plotted. Blue arrows indicate the length $R_{s}$ and orientation $\Theta$ of an angular mean vector (Methods and Supplementary Figure 1e) ${ }^{7}$. c, d Top-view (c) and side-view (d) of cells expressing AIP1-GFP (left) and D $\alpha$-cat-TagRFP (right) at $24 \mathrm{~h}$ APF. The vertical section along the dashed arrow in panel $\mathbf{c}$ is shown in $\mathbf{d}$. Alphabets indicate corresponding coordinates in different views. e, $\mathbf{g}$ Selected snapshots from movies of AIP1-GFP (gray in top panels, green in bottom panels) and D $\alpha$-cat-TagRFP (red in bottom panels). Blue arrowheads indicate remodeling (e) and stable (g) junctions. $\mathbf{f}$, $\mathbf{h}$ Plot of AIP1-GFP signal intensity (green, left $y$-axis) and length (blue, right $y$-axis) of AP junctions undergoing cell rearrangement (f) or not $(\mathbf{h}) . \ln \mathbf{f}, t=0$ indicates the time point when the junction was reconnected. Data shown in $\mathbf{h}$ indicate that bleaching was negligible. i Snapshots of utrABD-GFP before and after photobleaching (square indicates ROI). $t=0$ indicates the time point when photobleaching was conducted. $\mathbf{j}, \mathbf{k}$ utrABD-GFP in ROls examined before and after photobleaching along the AP (j) and PD (k) junctions. Color maps are shown in the upper right. I The stable fraction of utrABD-GFP. Welch's $t$-test: AP vs. PD, $P<0.01$. The number of junctions examined is indicated $(\mathbf{b}, \mathbf{f}, \mathbf{h}, \mathbf{I})$. Data are presented as the mean \pm s.e.m. (b) and as the mean \pm s.d. (f, $\mathbf{h}, \mathbf{I})$. Scale bars: $5 \mu \mathrm{m}(\mathbf{a}, \mathbf{e}, \mathbf{g}), 2 \mu \mathrm{m}$ $(\mathbf{c}, \mathbf{d}, \mathbf{i})$, and $0.5 \mu \mathrm{m}(\mathbf{j})$
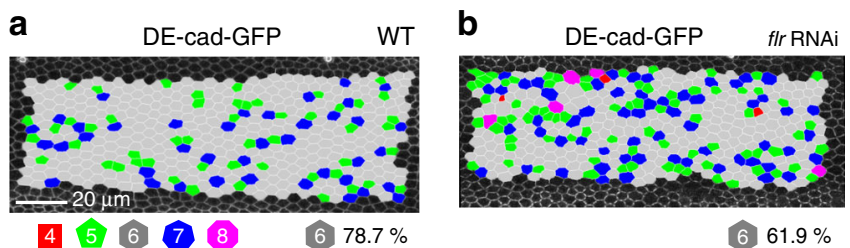

6. $61.9 \%$

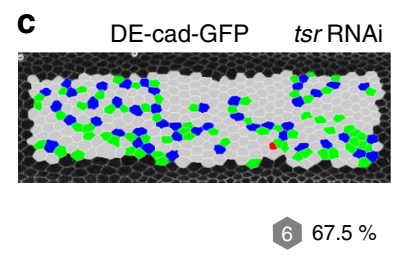

g

e

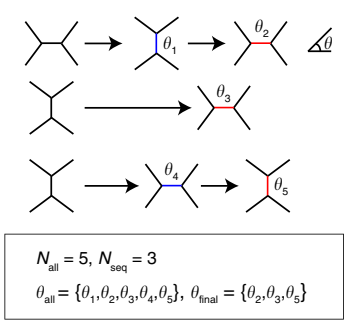

f

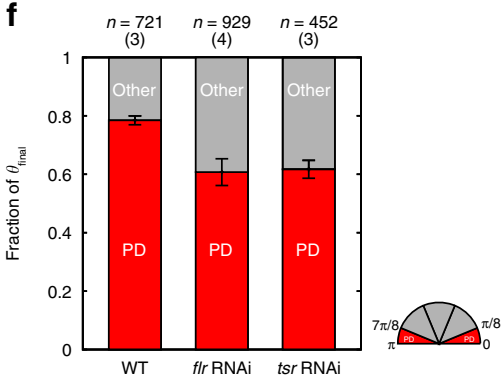

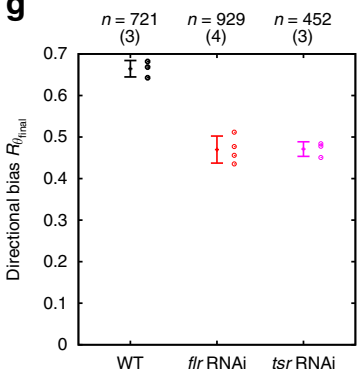

d

\begin{tabular}{|c|cc|}
\hline & \multicolumn{2}{|c|}{$\begin{array}{c}\text { Fraction of hexagonal cells } \\
\text { mean } \pm \text { s.d. }(\%)\end{array}$} \\
\hline WT $\left(25^{\circ} \mathrm{C}\right)$ & $75.6 \pm 3.1$ & $(n=10)$ \\
\hline fir $\mathrm{RNAi}$ & $63.0 \pm 4.1$ & $(n=10)$ \\
\hline WT $\left(29^{\circ} \mathrm{C}\right)$ & $74.8 \pm 2.3$ & $(n=11)$ \\
\hline tsr $\mathrm{RNAi}$ & $64.5 \pm 4.4$ & $(n=10)$ \\
\hline
\end{tabular}

h

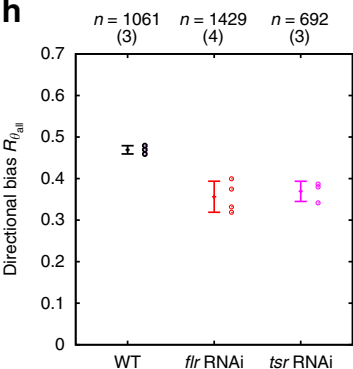

Fig. 2 AIP1 and cofilin are required for the efficient hexagonal cell packing and directional cell rearrangement. a-c Images of DE-cad-GFP with the indicated genotypes (a WT at $32 \mathrm{~h} \mathrm{APF}$; b fir RNAi at $32 \mathrm{~h} \mathrm{APF}$; and $\mathbf{c}$ tsr RNAi at $28 \mathrm{~h} \mathrm{APF}$ at $29{ }^{\circ} \mathrm{C}$, which corresponds to $\sim 32 \mathrm{~h}$ APF at $25{ }^{\circ} \mathrm{C}$ ). Cells are colored according to the number of junctions (red, square; green, pentagon; gray, hexagon; blue, heptagon; and magenta, octagon). d Table of the fraction of hexagonal cells for each indicated genotype. Welch's $t$-test: WT ( $25^{\circ} \mathrm{C}$ ) vs. flr RNAi, $P<0.001$, WT $\left(29^{\circ} \mathrm{C}\right.$ ) vs. tsr RNAi, $P<0.001$. e Schematic of cell rearrangement analysis. We tracked individual junctions that appeared in a movie and measured their angle relative to the PD axes $\left(\theta_{\mathrm{i}}\right)$ of newly generated junctions based on cell rearrangement. $\theta_{\text {final }}$ and $\theta_{\text {all }}$ are the sets of angles from the final round and all rounds of cell rearrangement, respectively, from which the magnitude of directional bias $R_{\theta f i n a l}$ and $R_{\theta \text { all }}$ were calculated (METHODS). $N_{\text {seq }}$ and $N_{\text {all }}$ indicate the numbers of remodeled junctions and cell rearrangements, respectively. f-h Quantification of cell rearrangement for each genotype based on time-lapse data captured at $24-27 \mathrm{~h}$ APF at $25^{\circ} \mathrm{C}$ (WT, flr RNAi) and time-lapse data acquired at $20.5-23 \mathrm{~h} 20 \mathrm{~m} \mathrm{APF}$ at $29^{\circ} \mathrm{C}$ (tsr RNAi). f PD (red) and other (gray) fractions of $\theta_{\text {final }}$. Classification of $\theta_{\text {final }}$ is illustrated with a semicircle. $\mathbf{g}$, $\mathbf{h} R_{\theta f i n a l}(\mathbf{g})$ and $R_{\theta \text { all }}(\mathbf{h})$ of each genotype. $\mathbf{g}$ Dunnett's test: WT vs. flr RNAi, $P<0.001$, WT vs. tsr RNAi, $P<0.001$. h Dunnett's test: WT vs. flr RNAi, $P<0.01$, WT vs. tsr RNAi, $P<0.01$. The number of pupae (d), and the numbers of events and pupae examined (f-h) are indicated. Data are presented as the mean \pm s.d. (d, f-h). Scale bar: $20 \mu \mathrm{m}$ (a)

localized at AP junctions following the l-o-f of two core PCP components, prickle $(p k)^{44}$ and flamingo/starry night ( $\mathrm{fmi} /$ stan) 45,46 (Fig. 3i-m), indicating that PCP is not required for correct localization of AIP1.

AIP1-cofilin are required for efficient cell rearrangement. Next, we examined whether and how the depletion of AIP1 affects directional cell rearrangement. We expressed dsRNA against $f l r$ using the Gal4-UAS system combined with a temperature shift (Supplementary Note 2). Under experimental conditions, GFPtagged endogenous AIP1 was not detected in flr RNAi cells (Supplementary Figure 2d-f). We also confirmed that flr RNAi did not alter tissue stress anisotropy per se (Supplementary Figure 4; Dunnett's test, $P>0.9)^{47}$. The angles of new junctions generated by cell rearrangement were measured in time-lapse movies obtained at 24-27 h APF. When junctions underwent multiple rounds of remodeling, final and preceding rounds of junctional remodeling were considered separately for analysis ( $\theta_{\text {final }}$ and $\theta_{\text {all }}$, and $N_{\text {seq }}$ and $N_{\text {all }}$ in Fig. 2e; METHODS). This is because the former affects the steady alignment of hexagonal cells along the PD axis that accelerates the formation of a hexagonal pattern (Supplementary Figure 1d), whereas the latter is an intermediate process of searching for an efficient relaxation pathway ${ }^{7,24}$. flr RNAi did not lower the frequency of cell rearrangement (Supplementary Figure 5; Dunnett's test, $P>0.05$ for $N_{\text {seq }} / N_{\text {cell }}$ and $P>0.05$ for $\left.N_{\text {all }} / N_{\text {cell }}\right)$. Both $\theta_{\text {final }}$ and $\theta_{\text {all }}$ became less biased toward the PD axis following flr RNAi, and the difference between WT and flr RNAi was larger for $\theta_{\text {final }}$ than for $\theta_{\text {all }}$ (Fig. 2f-h; Dunnett's test, $P<0.001$ for $R_{\theta f i n a l}$ and $P<0.01$ for $R_{\theta a l l} ;$ semicircle in Fig. $2 \mathrm{f}$ shows angle classification). Contractionelongation was affected by flr RNAi as expected from disoriented cell rearrangement (Supplementary Figure 6a, b).

As AIP1 is a cofactor of cofilin, we examined whether cofilin is involved in regulating AIP1 localization and PD cell rearrangement. In in vitro studies, the binding affinity of AIP1 for F-actin 
a

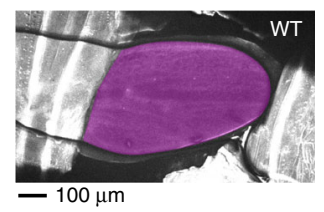

C
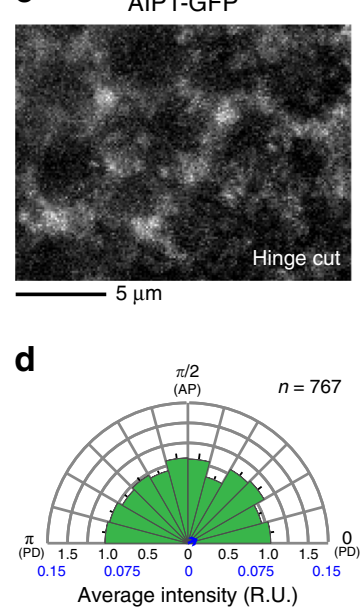

$R_{s}$

g

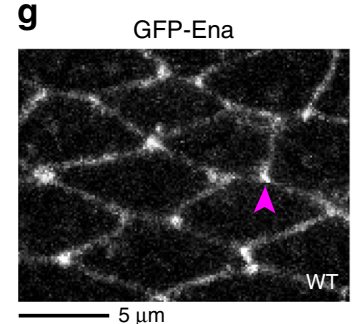

h

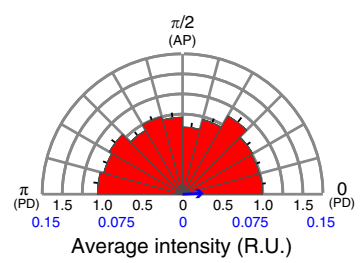

$R_{s}$

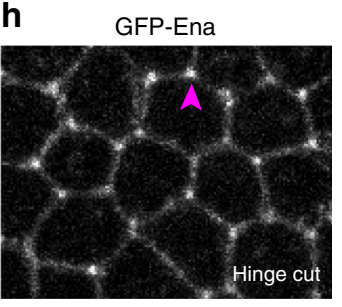

i
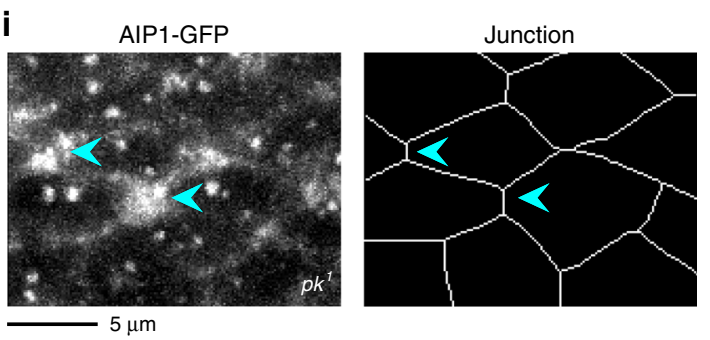

j

$R_{s}$
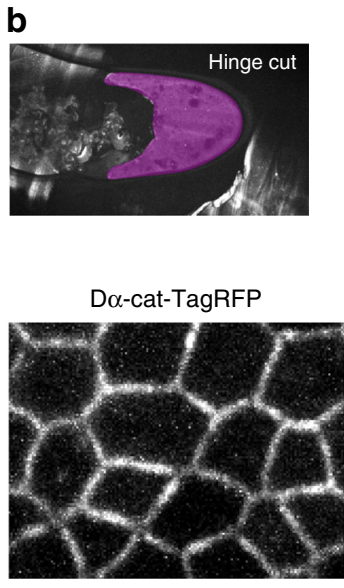

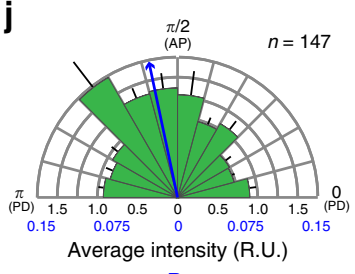

f

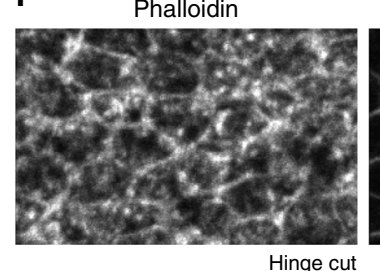

Hinge cut

k
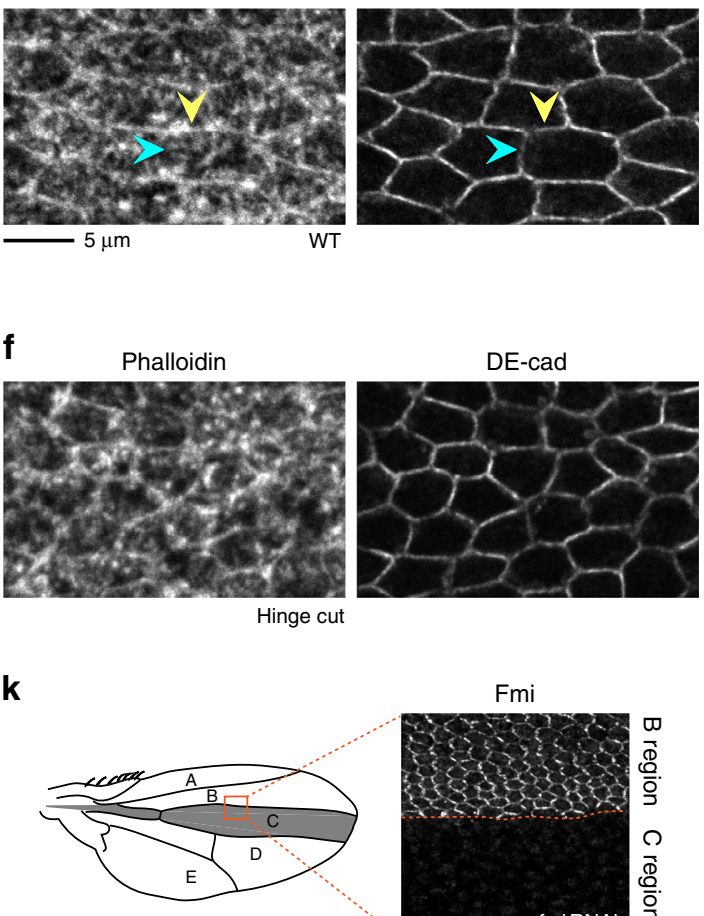

WT

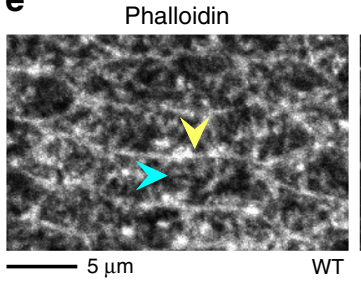

(
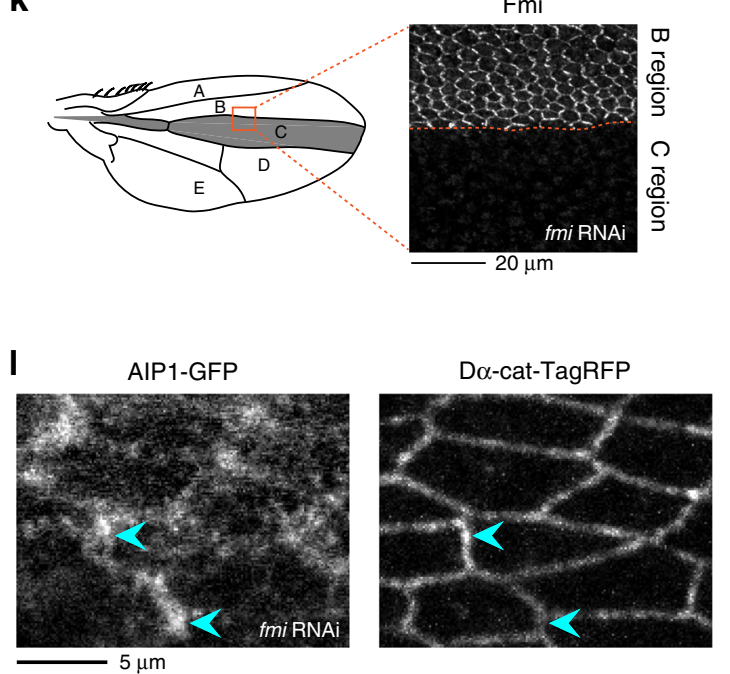

m

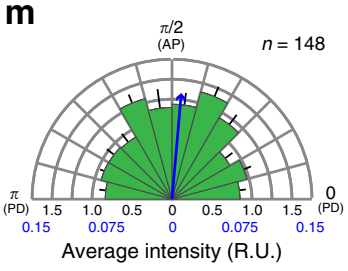

$R_{s}$

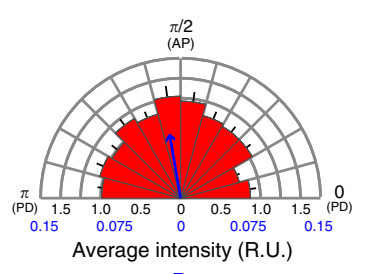

$R_{s}$ is much weaker in the absence of cofilin ${ }^{30}$, and cofilin binds to actin filaments for a longer period of time when F-actin severing is suppressed through the loss of cofactor proteins, including $\mathrm{AIP}^{31}$. In S. cerevisiae, cofilin hypomorph mutation leads to the diffusive distribution of AIP1 in the cytosol, whereas aip1 null mutation moderately alters the subcellular localization of cofilin $^{35}$. We determined whether these phenomena were also observed in the Drosophila pupal wing. In $t s r$ dsRNA-expressing cells, in which GFP-tagged endogenous cofilin fluorescence was not detected (Supplementary Figure 2j), AIP1 was diffusely distributed in the AJ plane, with little accumulation at the AJ plane along the apico-basal axis (Supplementary Figure 3b-e). flr dsRNA-expressing cells exhibited abnormal aggregates of cofilin in the cytosol (Supplementary Figure. 3f-k). The enrichment of cofilin at the junction is more evident along the plane of $\mathrm{AJ}$, presumably because cofilin remained in actin cables along the AJ 
Fig. 3 AIP1 localizes to AP junctions in a tissue stretch-dependent manner. $\mathbf{a}$, b Low magnification images of DE-cad-GFP of the WT (a) and mechanically relaxed (b) wings at $24 \mathrm{~h}$ APF. The wing blade is shaded magenta. c AIP1-GFP (left) and D $\alpha$-cat-TagRFP (right) in the mechanically relaxed wing at $24 \mathrm{~h}$ APF. d Directional bias of AIP1-GFP signal intensity (left) and D $\alpha$-cat-TagRFP signal intensity (right) in the mechanically relaxed wing was quantified as described for Fig. 1b. e, f F-actin distribution in WT (e) and mechanically relaxed (f) wings at $24 \mathrm{~h}$ APF. Phalloidin (left) and DE-cad (right). Yellow and blue arrowheads indicate PD and AP junctions, respectively. $\mathbf{g}$, $\mathbf{h}$ GFP-Ena in WT (g) and mechanically relaxed (h) wings at $24 \mathrm{~h}$ APF. Magenta arrowheads indicate the accumulation of GFP-Ena on cell vertices. $\mathbf{i}$ AIP1-GFP (left) in a $p k^{1}$ wing at $24 \mathrm{~h}$ APF. Cell contours are depicted (right). Blue arrowheads indicate AP junctions. $\mathbf{j}$ Directional bias of AIP1-GFP signal intensity in a pk $k^{1}$ wing. $\mathbf{k}$ Left: Schematics of the wing. The expression domain of ptc-Gal4 was shaded gray. A square indicates the region shown in right. Right: The Fmi antibody signal in a fmi RNAi wing at $24 \mathrm{~h}$ APF. I AIP1-GFP (left) and D $\alpha$-catTagRFP (right) in a fmi RNAi wing at $24 \mathrm{~h} \mathrm{APF.} \mathrm{Blue} \mathrm{arrowheads} \mathrm{indicate} \mathrm{AP} \mathrm{junctions.} \mathrm{The} \mathrm{animal} \mathrm{was} \mathrm{carrying} \mathrm{one} \mathrm{copy} \mathrm{of} \mathrm{AIP1-GFP,} \mathrm{and} \mathrm{the} \mathrm{brightness-}$ contrast was adjusted using different parameters compared to those used in other homozygous samples. m Directional bias of AIP1-GFP signal intensity (left) and D $\alpha$-cat-TagRFP signal intensity (right) in a fmi RNAi wing. The number of junctions examined is indicated (d, $\mathbf{j}, \mathbf{m}$ ). Data are presented as the mean \pm s.e.m. $(\mathbf{d}, \mathbf{j}, \mathbf{m})$. Scale bars: $100 \mu \mathrm{m}(\mathbf{a}), 5 \mu \mathrm{m}(\mathbf{c}, \mathbf{e}, \mathbf{g}, \mathbf{i}, \mathbf{I})$, and $20 \mu \mathrm{m}(\mathbf{k})$

a

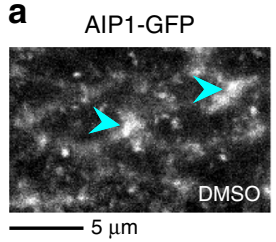

d

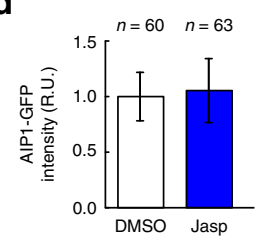

D $\alpha$-cat-TagRFP

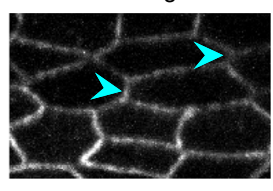

e

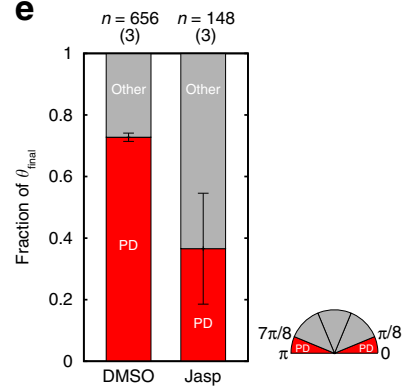

b AIP1-GFP

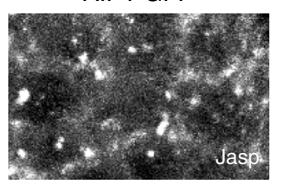

D $\alpha$-cat-TagRFP

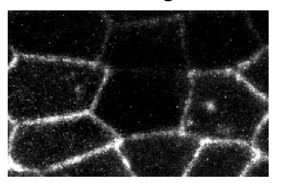

c

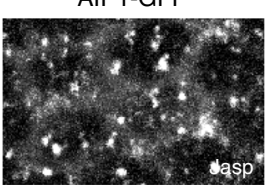

f

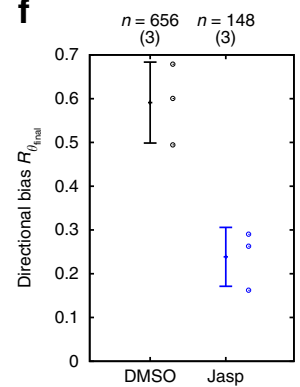

g $\quad n=954 \quad n=194$

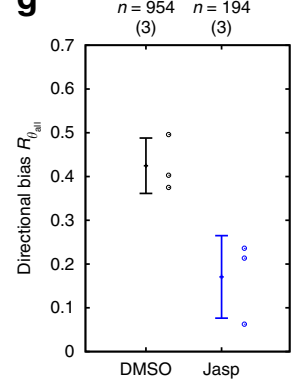

Fig. 4 Jasp treatment results in disoriented cell rearrangement. a-c AIP1-GFP (left) and D $\alpha$-cat-TagRFP (right), in DMSO-treated (a) and Jasp-treated $(\mathbf{b}, \mathbf{c})$ wings at $24 \mathrm{~h} \mathrm{APF}$. Images in panels $\mathbf{b}$, c show different regions of the same wing. Cells showing a faint signal of D $\alpha$-cat-TagRFP in $\mathbf{b}$ were located near the boundary of the ptc-Gal4 expressing region. Blue arrowheads indicate AP junctions. $\mathbf{d}$ The average signal intensity of AIP1-GFP along junctions in DMSO- and Jasp-treated wings. Welch's $t$-test: DMSO vs. Jasp, $P>0.2$. e-g Cell rearrangement defects induced by Jasp treatment were quantified as shown in Fig. 2e-h. e PD (red) and other (gray) fractions of $\theta_{\text {final. }} \mathbf{f}, \mathbf{g} R_{\theta \text { final }}(\mathbf{f})$ and $R_{\theta a l l}(\mathbf{g})$ for each condition. $\mathbf{f}$ Welch's $t$-test: DMSO vs. Jasp, $P<0.01$. $\mathbf{g}$ Welch's $t$-test: DMSO vs. Jasp, $P<0.05$. The number of junctions (d), and the numbers of events and pupae examined (e-g) are indicated. Data are presented as the mean \pm s.d. $(\mathbf{d}-\mathbf{g})$. Scale bar: $5 \mu \mathrm{m}$ (a)

plane for a longer period of time. Collectively, AIP1 and cofilin are mutually dependent for their localization, and phenotype quality and strength were conserved between fly and yeast. Next, we addressed the requirement for cofilin in directional cell rearrangement. tsr RNAi, without changing the inferred tissue stress anisotropy (Supplementary Figure 4; Dunnett's test, $P>$ 0.9 ), caused defects in hexagonal cell packing (Fig. 2c, d; Welch's $t$-test, $P<0.001$ ), directional cell rearrangement (Fig. $2 \mathrm{f}-\mathrm{h}$; Dunnett's test, $P<0.001$ for $R_{\theta \text { final }}$ and $P<0.01$ for $\left.R_{\theta \text { all }}\right)$, and contraction-elongation (Supplementary Figure $6 \mathrm{c}$ ), similar to that caused by flr RNAi.

The acute delocalization of AIP1 impairs cell rearrangement. We also pharmacologically inhibited the functions of AIP1/cofilin using the F-actin stabilization drug Jasplakinolide (Jasp). Jasp stabilizes F-actin by competitively inhibiting cofilin binding to Factin and decreasing the monomer off rate at filament ends ${ }^{48,49}$. As expected from the uniform distribution of AIP1 in $t s r$ RNAi cells (Supplementary Figure 3c), the biased distribution of AIP1 was lost in wings incubated with Jasp for $4 \mathrm{~h}$ (Fig. $4 \mathrm{a}-\mathrm{c}$ ). In contrast, the average signal intensity of AIP1-GFP along junctions was not significantly affected (Fig. $4 \mathrm{~d}$; Welch's $t$-test, $P>0.2$ ). Under these conditions, the directionality of cell rearrangement was weakened (Fig. 4e-g; Welch's $t$-test, $P<0.01$ for $R_{\theta \text { final }}$ and $P$ $<0.05$ for $\left.R_{\theta \mathrm{all}}\right)$, and the overall extent of contraction-elongation was decreased (Supplementary Figure 6d, e). In summary, the acute delocalization of AIP1 resulted in disoriented cell rearrangement, highlighting the importance of the subcellular localization of AIP1 in regulating directional cell rearrangement and suggesting that $\mathrm{flr}$ l-o-f phenotypes are unlikely to reflect early developmental defects.

Thus far, we showed that tissue tension specifies AIP1 localization in a cell, and AIP1 and its cofactor cofilin are required for efficient directional cell rearrangement. Thus, we next undertook experiments to answer three questions. First, how do AIP1 and cofilin promote directional cell rearrangement? Second, whether and how do AIP1 and cofilin facilitate a resistance to tissue tension during directional cell rearrangement? Third, how are AIP1 localization and function related to tissue tension?

Fmi and Dsh polarities are largely normal in flr RNAi cells. Previous studies reported that hexagonal cell packing is regulated through the PCP pathway via cadherin trafficking in the Drosophila pupal wing, and AIP1 is required for PCP establishment ${ }^{34,37,50}$. Thus, AIP1 potentially controls cell 


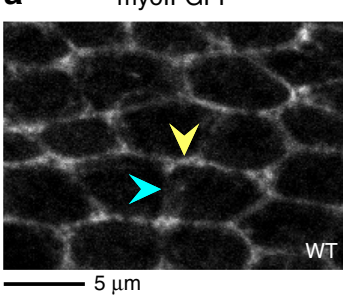

b

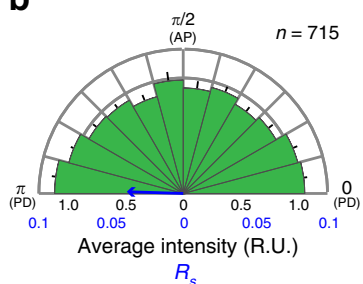

Do-cat-TagRFP

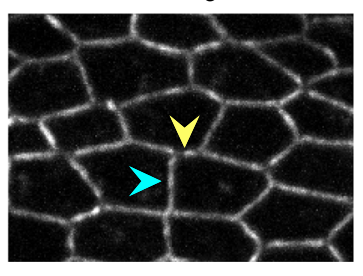

C

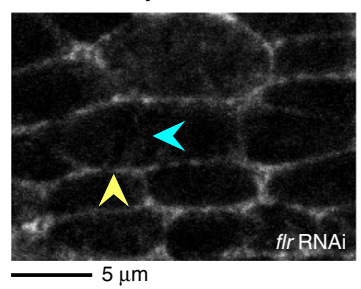

D $\alpha$-cat-TagRFP

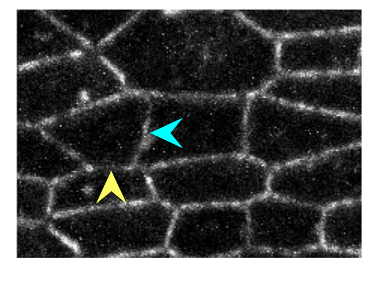

d

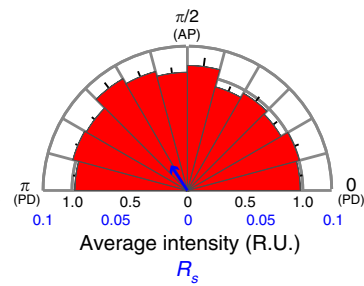

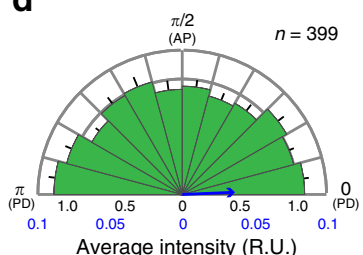

$R_{s}$

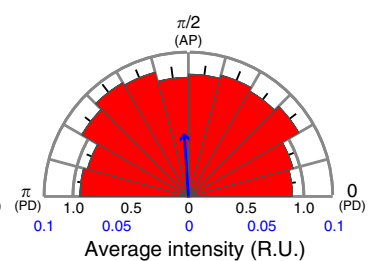

$R_{s}$

Fig. 5 The global myo-II polarization pattern is not affected by flr RNAi. a, c myo-II-GFP (left) and the AJ marker D $\alpha$-cat-TagRFP (right) in WT (a) and flr RNAi (c) wings at $24 \mathrm{~h}$ APF. Yellow and blue arrowheads indicate PD and AP junctions, respectively. b, d Directional bias of myo-II-GFP signal intensity (left) and D $\alpha$-cat-TagRFP signal intensity (right) along junctions in WT (b) and flr RNAi (d) wings was quantified as have been done in Fig. 1b. The number of junctions examined $(\mathbf{b}, \mathbf{d})$ is indicated. Data are presented as the mean \pm s.e.m. (b, d). Scale bars: $5 \mu$ m $(\mathbf{a}, \mathbf{c})$

rearrangement via the PCP pathway in the wing. However, we observed the enrichment of Fmi and Disheveled (Dsh) ${ }^{51}$ at AP (vertical) junctions in flr RNAi cells, similar to WT cells (Supplementary Figure $7 \mathrm{~b}, \mathrm{~d}, \mathrm{f}, \mathrm{h}$ ). To quantitatively evaluate these observations, we measured the orientation of Fmi and Dsh polarities in each cell using PCP nematics (Supplementary Figure $7 \mathrm{a})^{6}$. According to this analysis, an off-axis fraction was detected for the Fmi polarity of flr RNAi cells (Supplementary Figure 7c, e; Watson's test, $P<0.001$ ), and Dsh polarity distribution was indistinguishable between WT and flr RNAi (Supplementary Figure 7g, i; Watson's test, $P>0.4$ ). The discrepancy with previous studies may result from different l-o-f conditions employed (see Supplementary Note 2 for details).

To address how the Fmi polarity map affects cell rearrangement, we transiently overexpressed fmi-GFP, which has been shown to be a functional allele ${ }^{52}$, and measured the orientation of cell rearrangement in such tissues. fmi-GFP overexpression abolished Fmi polarity (Supplementary Figure 8a, b; Watson's test, $P<0.001$ ), whereas the directionality of cell rearrangement was only mildly affected compared with that of flr RNAi cells (Supplementary Figure 8c-e; Welch's $t$-test, $P>0.2$ for $R_{\theta \text { final }}$ and $P<0.01$ for $\left.R_{\theta \text { all }}\right)$. From these results, we concluded that cell rearrangement defects observed in the flr RNAi wing is unlikely to reflect PCP signaling dysfunction.

The detachment of myosin cables along remodeling junctions. We next focused on myo-II, a well-established regulator of cytoskeletal dynamics during junctional remodeling $22-25$. Previously, we showed that myo-II is localized on PD junctions in response to tissue stretching ${ }^{7}$. This global myo-II polarization pattern was not affected by flr RNAi (Fig. 5). However, a local structural change in junctional actomyosin was observed during cell rearrangement. myo-II formed a small ring-like structure, accompanied by $\mathrm{D}$ - $\alpha$-catenin (D- $\alpha$-cat) signal loss, in the short junctions of WT cells (Fig. 6a; hereafter called the myo-II ring), and much larger myo-II rings were detected in flr or tsr RNAi cells (Fig. 6b, c, j; Steel test, WT vs. flr RNAi, $P<0.001$, WT vs. $t s r$ RNAi, $P<0.001$ ). The septate junction (SJ) protein Discs large (Dlg) was present along short junctions (Fig. 6d-f), indicating that the myo-II ring structure did not represent cell extrusion; instead, it is generated by the detachment of myosin cables around the cell vertex (a similar subcellular structure was reported in ref. ${ }^{53}$ ), and the larger myo-II ring may represent its precocious formation. Time-lapse analysis showed that the myoII ring was only transiently formed immediately prior to the reconnection of cell contact surfaces in WT cells. In contrast, the myo-II ring was present for a longer duration in flr RNAi cells and occasionally failed to convert into stable junctions, potentially resulting in disoriented cell rearrangement (Fig. 61-n).

Mechanical rescue of myo-II ring enlargement. Interestingly, myo-II ring enlargement in flr RNAi cells was partially rescued by relaxing tissue stretch beginning at $15 \mathrm{~h} \mathrm{APF}$, when the extrinsic force begins to stretch the wing (Fig. 7a, b; Steel-Dwass test, WT vs. flr RNAi, $P<0.001$, WT vs. $f l r$ RNAi + hinge cut, $P>0.4$, $f l r$ RNAi vs. flr RNAi + hinge cut, $P<0.001)$. This result suggests that AIP1 prevents the detachment of junctional actomyosin, which runs perpendicular to the direction in which the wing is stretched, by anisotropic tissue tension (Fig. 7c).

Actin turnover ensures mechanoresitance of junctions. To characterize the molecular mechanism by which AIP1 and cofilin regulate the reorganization of junctional actomyosin, we searched for molecules that connect actomyosin to the AJ in an AIP1/ cofilin-dependent manner. Canoe (Cno) (Drosophila Afadin), which maintains the linkage between the actin cytoskeleton and the AJ in epithelial cells ${ }^{12,53,54}$, is one such candidate. We observed the specific loss of Cno in short junctions following $\mathrm{flr}$ or tsr RNAi (Fig. 6g-i). Moreover, cno RNAi induced the detachment of junctional actomyosin in wing cells (Fig. 6k). Together, these data indicate that disruption of the Cno-mediated linkage between actomyosin and the AJ leads to precocious formation of the myo-II ring.

This observation is similar to that of a previous study in which the inhibition of actin turnover destabilized the attachment of the actomyosin network to AJs during apical cell constriction ${ }^{55,56}$. FRAP analysis consistently showed that AIP1 and cofilin were required for efficient actin turnover along AP junctions (Fig. 8ae; Steel test, WT vs. flr RNAi, $P<0.01$, WT vs. $t s r$ RNAi, $P<$ 0.001 ). In addition, ectopic F-actin accumulation, which may potentially reflect sites of impaired actin turnover, was detected in RNAi cells (Supplementary Figure 9). Application of the F-actin 
a
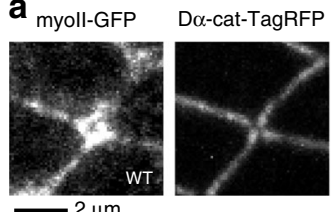

b $_{\text {myoll-GFP }}$
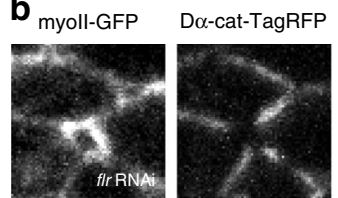

C myoll-GFP $_{\text {mat }}$

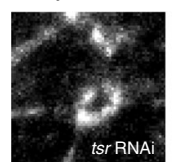

D $\alpha$-cat-TagRFP

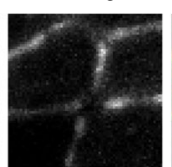

I
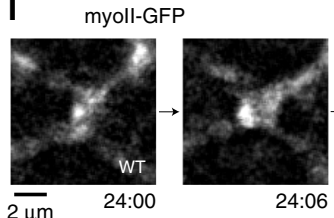

m myoll-GFP
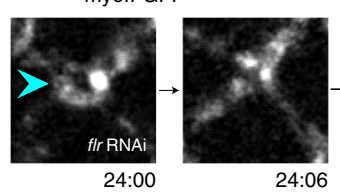
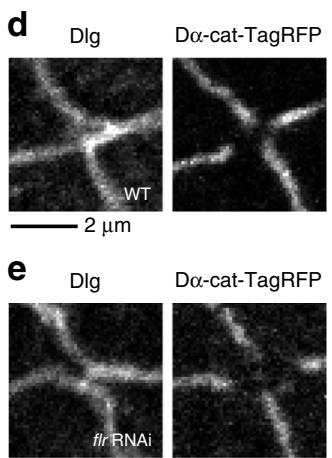

D $\alpha$-cat-TagRFP
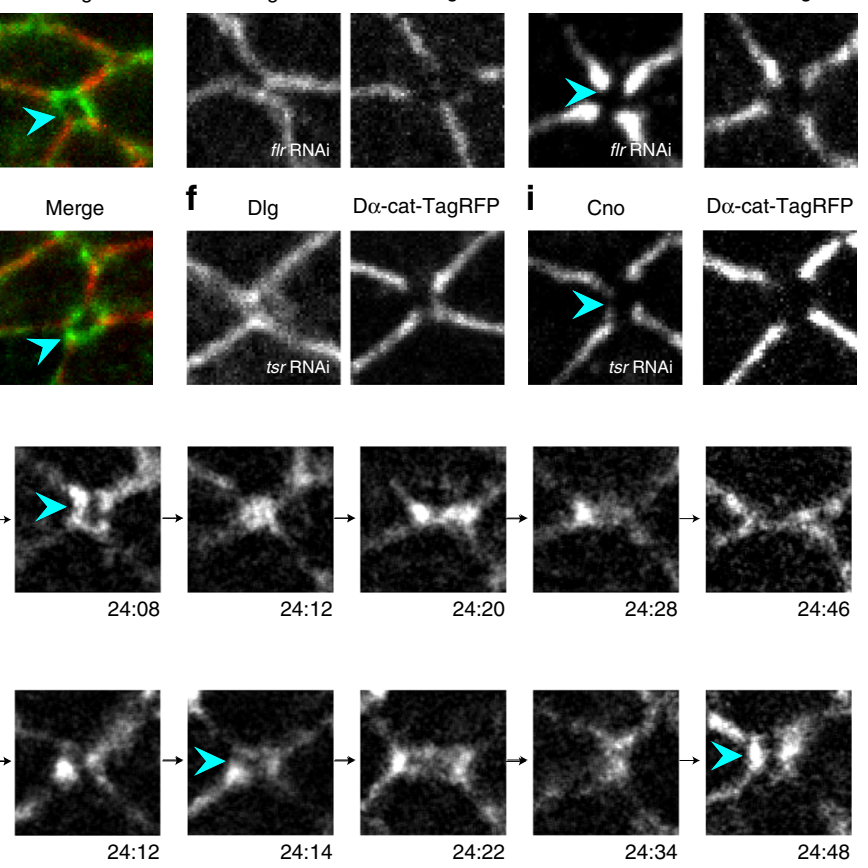
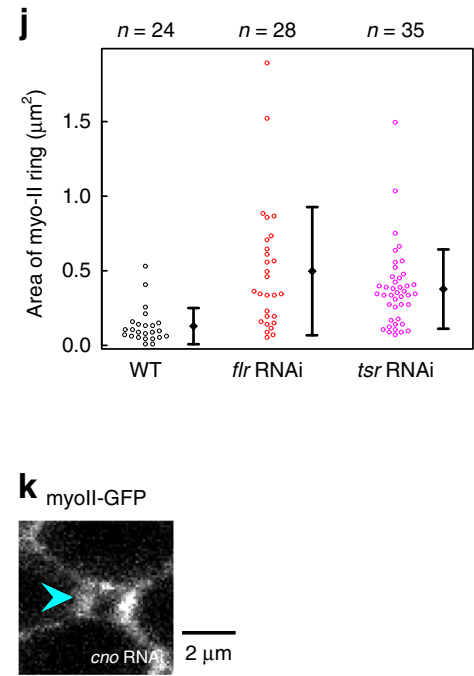

n

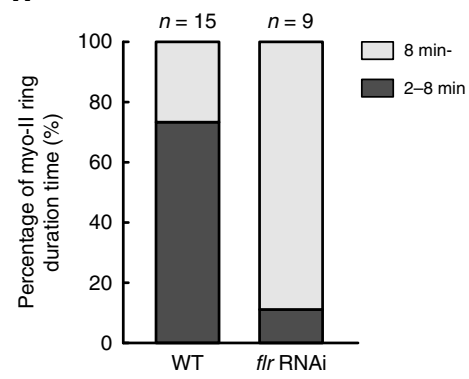

Fig. 6 flr or tsr RNAi disrupts the Cno-mediated linkage between actomyosin and the AJ in remodeling junctions. a Images of myo-II-GFP (gray in a left panel, green in a right panel) and D $\alpha$-cat-TagRFP (gray in a middle panel, red in a right panel) in a WT wing at $24 \mathrm{~h}$ APF. The blue arrowhead indicates a "myo-II ring" along the short remodeling junction. b, c Images of myo-II-GFP (gray in left panels, green in right panels) and D $\alpha$-cat-TagRFP (gray in middle panels, red in right panels) in a flr RNAi wing at $24 \mathrm{~h} \mathrm{APF} \mathrm{(b)} \mathrm{and} \mathrm{a} \mathrm{tsr} \mathrm{RNAi} \mathrm{wing} \mathrm{at} 20.5 \mathrm{~h} \mathrm{APF}$ at $29{ }^{\circ} \mathrm{C}$, which corresponds to $\sim 24 \mathrm{~h}$ APF at $25^{\circ} \mathrm{C}$ (c). myo-II rings were enlarged in RNAi cells (blue arrowheads). d-f Images of a SJ marker, Dlg (left), and D $\alpha$-cat-TagRFP (right) in WT (d), flr RNAi (e), and tsr RNAi (f) wings. The stages examined are described in a-c. Dlg was present in short junctions where D $\alpha$-cat-TagRFP signals were faint. $\mathbf{g}-\mathbf{i}$ Images of Cno (left) and D $\alpha$-cat-TagRFP (right) in WT (g), flr RNAi (h), and tsr RNAi (i) wings. The stages examined are described in a-c. flr or tsr RNAi resulted in the loss of Cno from short junctions (blue arrowheads). j The myo-Il ring area is plotted for each genotype. Steel test: WT vs. flr RNAi, $P<0.001$, WT vs. tsr RNAi, $P<0.001$. The differences between WT and RNAi groups were statistically significant after excluding outliers above $1.2 \mu m^{2}$. $\mathbf{k}$ An image of myo-IIGFP in a cno RNAi wing at $24 \mathrm{~h}$ APF. cno RNAi induced the detachment of junctional actomyosin (blue arrowhead). I, $\mathbf{m}$ Time-lapse images of myo-II-GFP in WT (I) and flr RNAi (m) wings. Blue arrowheads indicate myo-II rings. The myo-Il rings were not converted into stable PD junctions in flr RNAi cells. Note that GFP signal intensity was modified via image processing; thus, we only tracked structural changes in these images. $\mathbf{n}$ The fraction of myo-II ring time duration for each genotype. The number of ROls examined (j), and the number of events examined ( $\mathbf{n}$ ) are indicated. Data are presented as the mean \pm s.d. (j). Scale bars: $2 \mu \mathrm{m}(\mathbf{a}, \mathbf{d}, \mathbf{g}, \mathbf{k}, \mathbf{I})$

stabilization drug Jasp from 15 to $24 \mathrm{~h}$ APF induced myo-II ring enlargement and the loss of Cno from the short AP junctions (Fig. 8f, g, i-k). Furthermore, myo-II ring size returned to normal values following tissue stretch relaxation (Fig. 8h, i; Steel-Dwass test, DMSO vs. Jasp, $P<0.001$, DMSO vs. Jasp + hinge cut, $P>$ 0.8 , Jasp vs. Jasp + hinge cut, $P<0.001)$. Collectively, these data show that AIP1 and cofilin promote actin turnover to regulate the Cno-dependent reorganization of junctional actomyosin in a specific region and at the appropriate time during cell rearrangement. This mechanism is essential for cells to resist the mechanical load imposed on the remodeling AP junction in a process of complete directional cell rearrangement.

Cooperative cofilin binding links tissue tension and AIP1. Finally, we addressed how tissue tension is related to AIP1 localization and function (Fig. 9). Actin filaments alter their helical pitch in response to applied forces, and these structural changes are amplified by positive feedback between cofilin binding and F-actin twisting (Fig. 10a) ${ }^{15,17-19}$. This suggests that in the pupal wing, F-actin along the $\mathrm{PD}$ axis, which is under tension, adopts a less twisted configuration, leading to the preferential binding of cofilin and AIP1 to more twisted F-actin along the AP axis via positive feedback between cofilin binding and F-actin twisting. To examine this hypothesis, we took advantage of an actin mutant that inhibits cooperative cofilin binding to F-actin (yeast actin $\left.{ }^{G 146 V}\right)^{57}$. Following misexpression of the corresponding mutant Drosophila actin5C (act5C) gene, AIP1-GFP did not accumulate at AP junctions (Fig. 9a, b). In contrast, the global polarity of myo-II distribution was not significantly affected, although myo-II-GFP signals became diffuse along the junction (Fig. 9c, d). Moreover, the misexpression of mutant act5C caused the detachment of junctional actomyosin and the AJ (Fig. 9e, f; Wilcoxon rank sum test, $P<0.001$ ), resulting in impaired hexagonal cell packing (Fig. 9g-i; Welch's $t$-test, $P<0.001)$. These developmental defects were specifically 

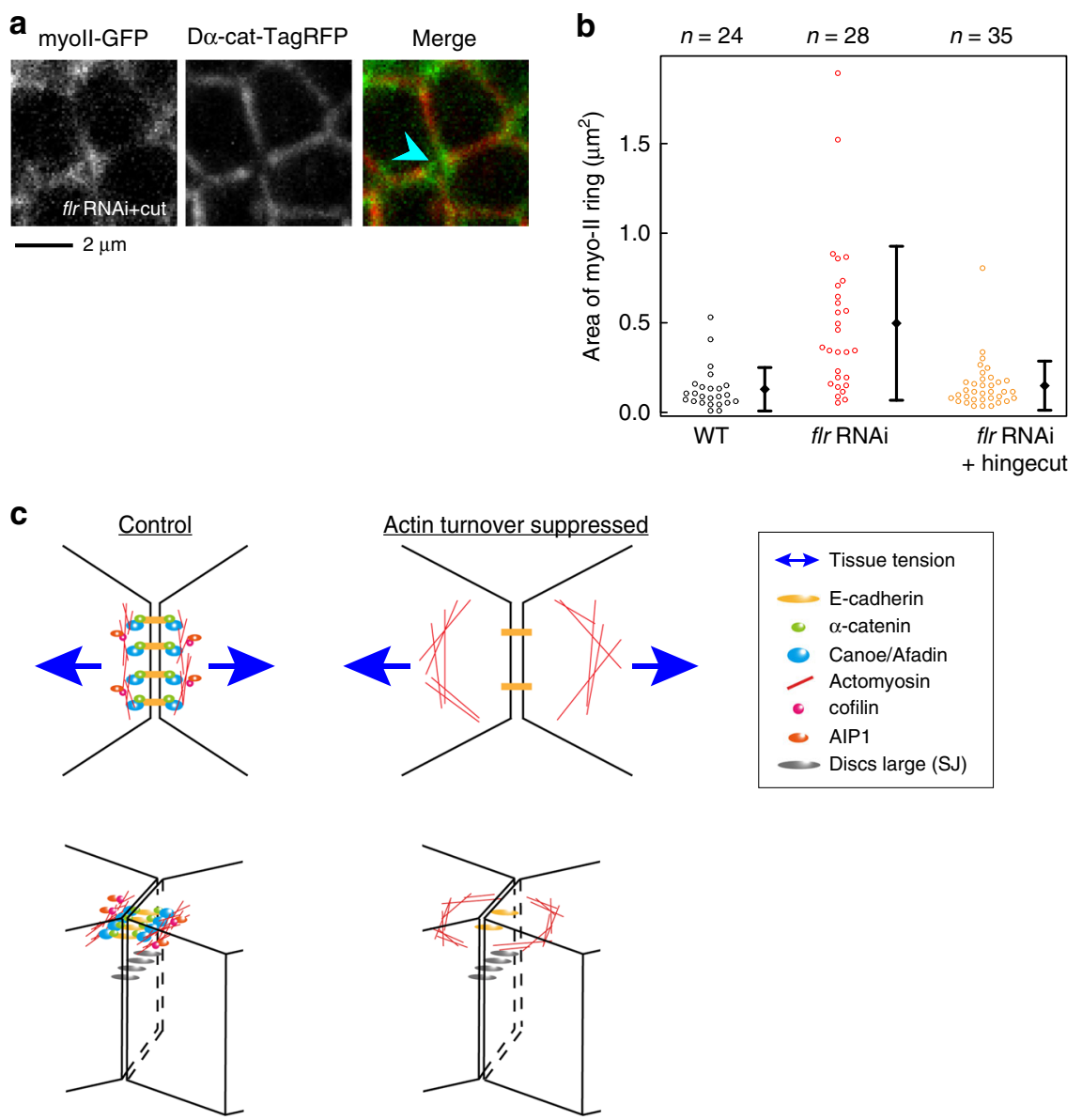

Fig. 7 myo-II ring enlargement in flr RNAi cells is rescued by relaxing tissue stretch. a Images of myo-II-GFP (gray in a left panel, green in a right panel) and D $\alpha$-cat-TagRFP (gray in a middle panel, red in a right panel) in a mechanically relaxed flr RNAi wing. The wing was detached from the hinge at $15 \mathrm{~h}$ APF and observed at $24 \mathrm{~h} \mathrm{APF}$. The blue arrowhead indicates a myo-II ring on the short remodeling junction. $\mathbf{b}$ The myo-II ring area is plotted for each genotype (WT and flr RNAi data were same as that shown in Fig. 6j). Steel-Dwass test: WT vs. flr RNAi, $P<0.001, W T$ vs. flr RNAi + hinge cut, $P>0.4$, flr RNAi vs. flr RNAi + hinge cut, $P<0.001$. The difference between flr RNAi and flr RNAi + hinge cut was statistically significant after excluding outliers above $1.2 \mu m^{2}$. c Schematic of the defects resulting from genetic or pharmacological manipulations to stabilize F-actin. AJ (E-cad) (yellow), $\alpha$-cat (green), Cno (light blue), actomyosin (red), cofilin (magenta), AIP1 (orange), Dlg (gray), and tissue tension generated by the extrinsic stretching force (blue arrows). Top (top) and side (bottom) views are shown. Left: In WT tissue, cells maintain AJ and actomyosin integrity along the AP junction in the presence of anisotropic tissue tension. Right: F-actin stabilization weakens Cno-mediated linkage between actomyosin and AJ. Subsequently, actomyosin detaches from the AP junction. The number of ROls examined is indicated (b). Data are presented as the mean \pm s.d. (b). Scale bar: $2 \mu \mathrm{m}$ (a)

induced by the mutant Actin but not its wild-type counterpart (Supplementary Figure 10). Thus, our data support the hypothesis that the cooperative binding of cofilin to twisted, mechanically relaxed F-actin links anisotropic tissue tension and AIP1 localization and function (Fig. 10b).

\section{Discussion}

In addition to global patterning determined by signaling molecules, global mechanical patterning represents another strategy for facilitating the long-range coordination of tissue development. This concept raises the questions as to how tissue-scale forces regulate biochemical signaling within or between cells and how cells subjected to tissue-scale forces maintain structural integrity when undergoing morphogenetic processes. The results of the present study highlight that actin regulation mediated through AIP1 and cofilin achieves an optimal balance between resistance to anisotropic tissue tension and morphogenesis.

The current working hypothesis (Fig. 10b) is that cells respond to anisotropy in tissue tension via the cooperative binding of cofilin to twisted F-actin, leading to AIP1 accumulation along remodeling AP junctions. AIP1 and cofilin facilitate a resistance to anisotropic tissue tension and maintain junction stability, thereby supporting PD cell rearrangement. Our observation that the misexpression of mutant act5C induced the delocalization of AIP1 and phenocopied flr RNAi is consistent with the current working hypothesis that postulates the positive feedback between cofilin binding and F-actin twisting. Future development of a biophysical method to visualize the helical pitch of actin filaments inside a cell will enable a more direct test.

We speculate that the myo-II ring is required to reconnect cell contact surfaces at four-way junctions. AIP1 and cofilin likely protect this temporally loosened junctional structure from anisotropic tissue tension by promoting actin turnover and strengthening the linkage between actomyosin and AJ via Cno, the Drosophila Afadin protein. Detailed molecular mechanisms that link actin turnover, junctional actomyosin, and E-cad/a-cat/ Afadin remain elusive. Rapid actin turnover can prevent the aggregation of a contractile actin network and thereby maintain its uniform network structure ${ }^{56,58}$, which may be required for stable binding of F-actin to E-cad/a-cat/Afadin. AIP1-mediated actin turnover can also activate biochemical signaling that 
a

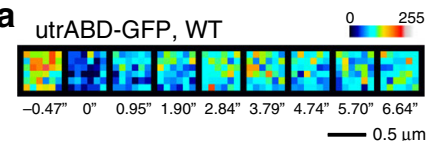

b

utrABD-GFP, flr RNAi $\quad 255$

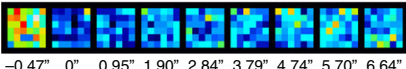

C utrABD-GFP, tsr RNAi $\quad 255$

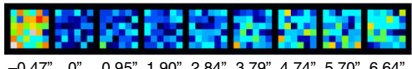

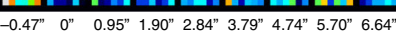
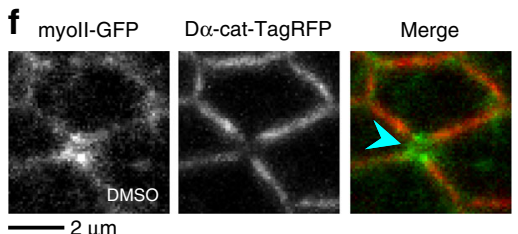

9 myoll-GFP
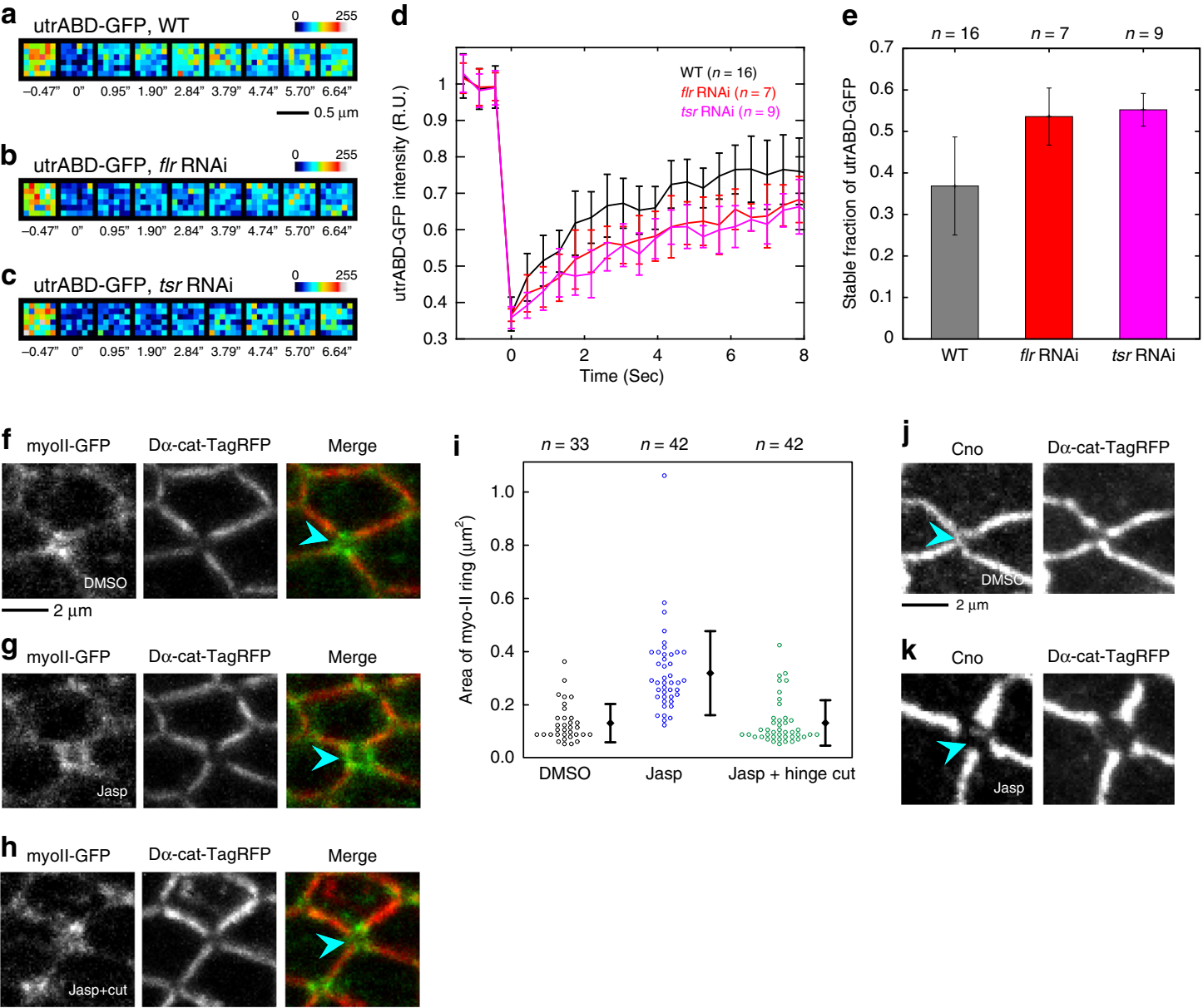

j

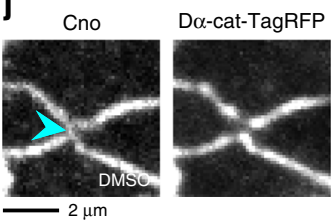

K Cno

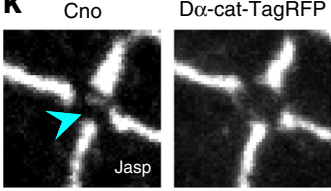

Fig. 8 Actin turnover underlies the reorganization of actomyosin and cell adhesion. a-c utrABD-GFP in ROls examined before and after photobleaching in the wings with the indicated genotypes (a WT at $24 \mathrm{~h} \mathrm{APF} ; \mathbf{b}$, flr RNAi at $24 \mathrm{~h} \mathrm{APF}$; and $\mathbf{c}$, tsr RNAi at $20.5 \mathrm{~h} \mathrm{APF}$ at $29^{\circ} \mathrm{C}$, which corresponds to $\sim 24 \mathrm{~h}$ APF at $25^{\circ} \mathrm{C}$ ). Color maps are shown in the upper right. d Recovery of utrABD-GFP signal intensity after photobleaching at AP junctions (black, WT; red, flr RNAi; magenta, tsr RNAi). utrABD-GFP signal intensity was normalized by its average value at three time points prior to photobleaching. The average of the data from individual junctions is plotted. e The stable fraction of utrABD-GFP for each genotype. Steel test: WT vs. flr RNAi, $P<0.01$, WT vs. tsr RNAi, $P<$ 0.001. f-h Images of myo-II-GFP (gray in left panels, green in right panels) and D $\alpha$-cat-TagRFP (gray in middle panels, red in right panels) in a DMSOtreated wing (f), Jasp-treated wing $(\mathbf{g})$, and Jasp-treated wing, which were detached from their hinges at $15 \mathrm{~h} \mathrm{APF} \mathrm{(h).} \mathrm{All} \mathrm{images} \mathrm{were} \mathrm{acquired} \mathrm{at} 24 \mathrm{~h}$ APF. Blue arrowheads indicate myo-II rings. $\mathbf{i}$ The myo-Il ring area is plotted for each condition. Steel-Dwass test: DMSO vs. Jasp, $P<0.001, \mathrm{DMSO}$ vs. Jasp + hinge cut, $P>0.8$, Jasp vs. Jasp + hinge cut, $P<0.001$. j, k Images of Cno (left) and D $\alpha$-cat-TagRFP (right) in DMSO-treated (j) and Jasp-treated (k) wings at $24 \mathrm{~h} \mathrm{APF}$. Blue arrowheads indicate the short AP junctions. The number of junctions examined (d, e) and the number of ROI examined (i) are indicated. Data are presented as the mean \pm s.d. $(\mathbf{d}, \mathbf{e}, \mathbf{i})$. Scale bars: $0.5 \mu \mathrm{m}(\mathbf{a})$ and $2 \mu \mathrm{m}(\mathbf{f}, \mathbf{j})$

controls linker proteins, including $\alpha$-cat and Afadin. In addition, it may potentially speed up lateral mobility of E-cad clusters to promote junctional remodeling ${ }^{36,59}$. Notably, candidate mechanisms mentioned above are not mutually exclusive. In the future, it would be necessary to address how different mechanisms act in concert to realize mechanoresistance of remodeling junctions.

This study uncovered a link between the biased distribution of AIP1 along the planar axis of a tissue and its function, a concept that was not addressed in previous studies investigating the role of AIP1 in tissue development ${ }^{33,34,36-38}$. Our data clearly indicate that tissue stretching is necessary for the AP-biased localization of AIP1; however, this observation also implies the existence of other factors because AIP1 accumulates specifically at remodeling AP junctions. Given that F-actin dynamically changes its distribution during development according to tissue tension patterns (Fig. 3e, $\mathrm{f}$ and our unpublished data), a more integrated understanding of actin dynamics and various ABPs is required to fully elucidate the regulatory mechanism underlying AIP1 localization.
In conclusion, the present study suggests two overlapping general strategies for the mechanical control of tissue development. First, tissue tension acts as a mechanical cue to bias protein localization and morphogenetic cell processes, thereby strengthening adjustability and flexibility to regulate development. The conversion of tissue tension into structural changes in the actin filament/network via $A B P$ interactions may be a conserved strategy for mechanotransduction ${ }^{60,61}$, although conclusive, direct evidence in vivo is required. Second, global mechanical patterning must be reconciled to maintain the structural integrity of the cell/tissue. Future studies should explore whether the molecular mechanisms identified in the present study potentially function in other developmental contexts, such as cell proliferation and apoptosis, and/or in other epithelial tissues.

\section{Methods}

Generation of transgenic flies. To construct pUAST-attB-act5C ${ }^{W T}$, pUAST-attB$a c t 5 C^{G 147 V}$, and pUAST-act5C $C^{G 147 V}$, act5C was PCR-amplified from a cDNA clone (Drosophila Genomics Resource Center \#LD18090). The G147V mutation was introduced using the GeneArt ${ }^{\circledR}$ Site-Directed Mutagenesis System (Thermo Fisher 
a

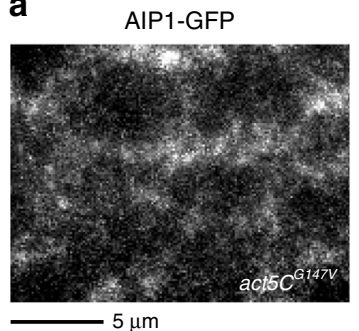

b

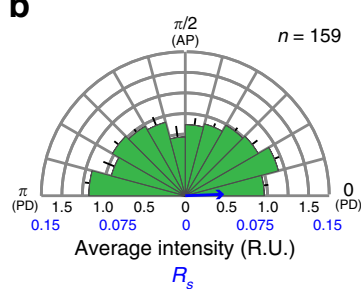

$R_{s}$
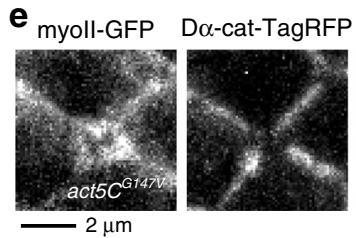

f

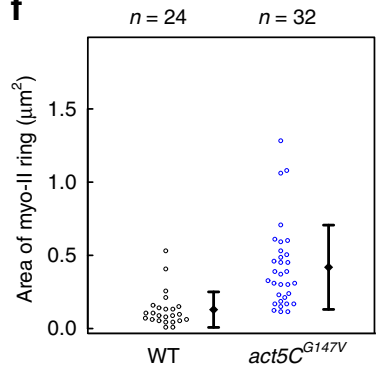

C
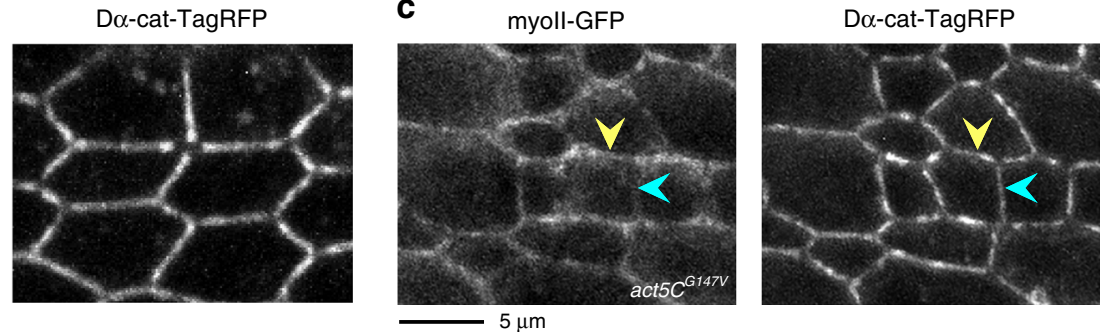

d

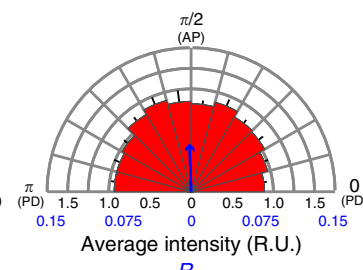

$R_{s}$

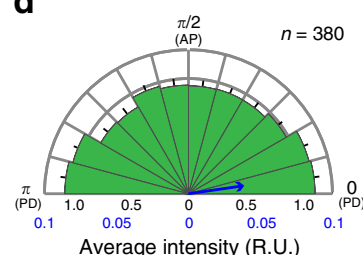

$R_{s}$

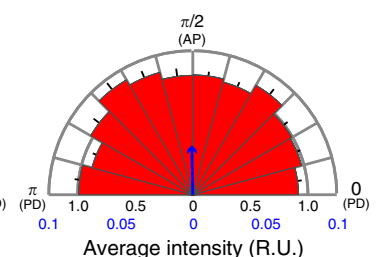

$R_{s}$

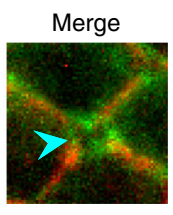

g

h
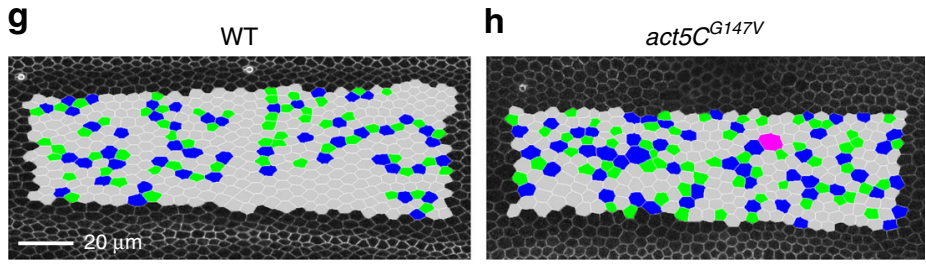

$\begin{array}{llllllll}4 & 5 & 6 & 7 & 8 & 6 & 74.2 \%\end{array}$

$62.2 \%$

Fig. 9 Cooperative binding of cofilin to F-actin is required for the biased distribution of AIP1, the integrity of junctional actomyosin, and hexagonal cell packing. a AIP1-GFP (left) and the AJ marker D $\alpha$-cat-TagRFP (right) in an act5CG147V misexpressed wing at $20.5 \mathrm{~h} \mathrm{APF}$ at $29^{\circ} \mathrm{C}$, which corresponds to $\sim 24$ $\mathrm{h}$ APF at $25^{\circ} \mathrm{C}$. b Directional bias of AIP1-GFP signal intensity (left) and D $\alpha$-cat-TagRFP signal intensity (right) along junctions in act5C $\mathrm{G} 147 \mathrm{~V}$ misexpressed cells was quantified as described for Fig. 1b. c myo-II-GFP (left) and the AJ marker D $\alpha$-cat-TagRFP (right) in an act5C G147V misexpressed wing at $20.5 \mathrm{~h}$ APF at $29^{\circ} \mathrm{C}$. Yellow and blue arrowheads indicate PD and AP junctions, respectively. $\mathbf{d}$ Directional bias of myo-II-GFP signal intensity (left) and D $\alpha$-cat-TagRFP signal intensity (right) along junctions in an act5CG147V misexpressed wing. e Images of myo-II-GFP (gray in a left panel, green in a right panel) and D $\alpha$-catTagRFP (gray in a middle panel, red in a right panel) in an act5CG147V misexpressed wing. Blue arrowhead indicates myo- $I$ ring. $\mathbf{f}$ myo- $I I$ ring area. Wilcoxon rank sum test: WT vs. act5CG147V, P<0.001. g, h Images of DE-cad-GFP with the indicated genotypes at $28 \mathrm{~h}$ APF at $29^{\circ} \mathrm{C}$, which corresponds to $\sim 32 \mathrm{~h}$ APF at $25^{\circ} \mathrm{C}$ (g, WT; h, act5CG147V misexpression). Cells are colored according to the number of junctions. $\mathbf{i}$ Table listing the fractions of hexagonal cells for each genotype. Welch's $t$-test: WT vs. act5CG147V,$P<0.001$. The number of junctions examined (b, d), the number of ROI examined (f), and the number of pupae (i) are indicated. Data are presented as the mean \pm s.e.m. (b, d) and as the mean \pm s.d. (f, i). Scale bars: $5 \mu \mathrm{m}(\mathbf{a}, \mathbf{c}), 2 \mu \mathrm{m}(\mathbf{e})$, and $20 \mu \mathrm{m}(\mathbf{g})$

Scientific) with the following primer: 5'-GCTTTCTCTCTACGCCTCCGTCCGT ACCACAGGTATCGTG-3'. Subsequently, act5C $C^{W T}$ or act5C $C^{G 147 V}$ was cloned into pUAST-attB or pUAST cut with EcoRI and NotI. The generated pUAST-attB and pUAST plasmids were injected into flies carrying $22 \mathrm{~A}$ or $86 \mathrm{Fa}$ attP landing sites ${ }^{62}$, or $y w$, respectively, to produce transgenic flies.

Inverse PCR. To determine an insertion site for the P-element containing an artificial exon encoding GFP in the tsr-GFP line (\#ZCL0613) ${ }^{40}$, we followed protocols by BDGP (http://www.fruitfly.org/about/methods/inverse.pcr.html) and by Hoskins and Evans-Holm (http://flypush.imgen.bcm.tmc.edu/pscreen/files/ GDP_iPCRProtocol_051611.pdf). Briefly, genomic DNA of the tsr-GFP line was extracted and subjected to restriction digestion using Sau3AI or HhaI, and the resultant DNA fragment was self-ligated. Then, the $3^{\prime}$ end of P-element and its flanking genomic DNA was amplified by using EY3.F and EY3.R primers for sequencing. The insertion site was determined using BLAST from FlyBase (http:// flybase.org/).
Western blotting. Pupae of $y w$, flareGFP, or $t s r G F P$ at $24-28 \mathrm{~h}$ APF were lysed in Laemmili's sample buffer (62.5 mM Tris (pH 6.8), 2\% SDS, $10 \%$ glycerol, $0.001 \%$ bromophenol blue, and $713 \mathrm{mM} \beta$-mercaptoethanol). The lysates were boiled for $20 \mathrm{~min}$ and these samples were loaded into a $10 \%$ poly-acrylamide gel. After SDSPAGE, samples were transferred to a PDMS membrane (Bio-Rad), blocked with $3 \%$ BSA-TBS (20 mM Tris (pH 7.2), $150 \mathrm{mM} \mathrm{NaCl}$ ) and incubated with anti-GFP (1/1000, SantaCruz sc-9996) or anti-a-tubulin (1/2000, MBL PM054) diluted in 3\% BSA-TBS overnight at $4{ }^{\circ} \mathrm{C}$. The membrane was washed with TBS- $0.05 \%$ tween 20 and incubated with HRP-conjugated secondary antibodies (ThermoFisher) diluted in 3\% BSA-TBS for at least $1 \mathrm{~h}$ at room temperature. Blots were developed using ECL Western Blotting Detection Reagents (GE healthcare) and imaged using LAS4000 IR multi color (FUJIFILM). An original scan of the western blot is shown in Supplementary Figure 11.

Drosophila genetics. Fly strains and a list of genotypes are summarized in Supplementary Methods. 
a

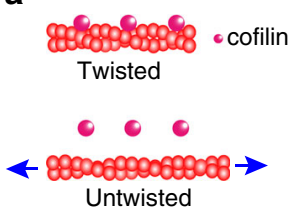

b

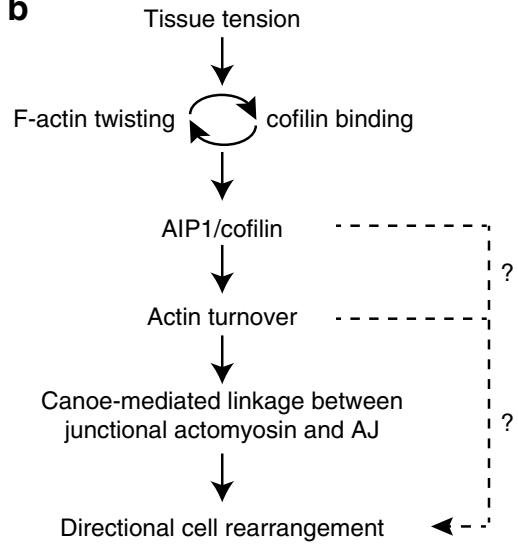

Fig. 10 Summary and working hypothesis. a Schematic showing tension on actin filaments and their twisting, which have been reported by previous studies using in vitro reconstituted actin filaments ${ }^{15,}$ 17-19. Actin filaments (red) alter their helical pitch in response to applied forces (blue arrows). cofilin (magenta) preferentially binds to twisted, mechanically relaxed actin filaments. b Summary of the working hypothesis. See the main text for details. Upper and bottom dashed arrows indicate an unidentified pathway regulating directional cell rearrangement downstream to AIP1/cofilin or actin turnover, which may potentially function via E-cad transport ${ }^{36}$

Immunohistochemistry and phalloidin staining. Anti-Cno (1/400) ${ }^{63}$, anti-Dlg (1/ $500)^{64}$, anti-Dsh $(1 / 1000)^{65}$, and anti-Fmi $(1 / 10)^{45}$ antibodies were used. Pupae at appropriate ages were dissected, and wings were fixed at room temperature for $30 \mathrm{~min}$ in PBS containing $4 \%$ paraformaldehyde. After washing with PBS containing $0.1 \%$ Triton X-100, these preparations were incubated overnight at $4{ }^{\circ} \mathrm{C}$ with the indicated antibodies.

To visualize F-actin in wing cells, dissected wings were incubated overnight at $4{ }^{\circ} \mathrm{C}$ with Alexa Fluor 546 Phalloidin (1/1000, invitrogen A22283). The condition of dissecting and fixing pupae was same as above.

Image collection. To prepare the Drosophila pupal wing samples for image collection, pupae at appropriate ages were fixed to double-sided tape and the pupal case above the left wing was removed. The pupae were then placed on a small drop of water or Immersol W 2010 (Zeiss 444969-0000-000) in a glass bottom dish with the left side facing downward $6,7,46,66$. The fixed time-point images other than Fig. 3a, b and time-lapse images shown in Fig. 6l, $\mathrm{m}$ were acquired using an inverted confocal microscope (A1R; Nikon) equipped with a $\times 60 /$ NA1.2 Plan Apochromat water-immersion objective at $25^{\circ} \mathrm{C}$. Other images were acquired using an inverted confocal spinning disk microscope (Olympus IX83 combined with Yokogawa CSU-W1) equipped with an iXon3 888 EMCCD camera (Andor), an Olympus $\times 60 / \mathrm{NA1} .2$ SplanApo water-immersion objective, and a temperature control chamber (TOKAI HIT), using IQ 2.9.1 (Andor) ${ }^{66}$. After imaging, we confirmed that the pupae survived to at least the pharate stage.

Surgical manipulation to relax the tissue stretch. Wings were detached from the hinges using forceps ${ }^{6,7}$. Hinges were severed at $23.5-24 \mathrm{~h}$ APF to analyze the effects of tissue tension on AIP1 localization (Fig. 3) or at $15 \mathrm{~h}$ APF for rescue experiments (Figs. 7, 8).

Jasplakinolide treatment. A small drop of Jasplakinolide (Jasp) (Invitrogen J7473) solution, which was diluted to $100 \mu \mathrm{M}$ or $200 \mu \mathrm{M}$ in $10 \%$ or $20 \%$ dimethyl sulfoxide (DMSO; Nacalai \#13408-64), was placed on a glass bottom dish. After removing the pupal case above the left wing, the pupae were placed in Jasp solution with the left side facing downward. Ten percent or $20 \%$ DMSO solution was used as the control. Filter papers soaked in DMSO or Jasp solution were placed along the pupae to prevent drying. For the acute treatment shown in Fig. 4, the pupae were incubated with $200 \mu \mathrm{M}$ Jasp solution starting at $20 \mathrm{~h}$ APF and observed at $24 \mathrm{~h}$ APF. For the experiments shown in Fig. 8, the pupae were incubated with $100 \mu \mathrm{M}$ Jasp solution starting at $15 \mathrm{~h} \mathrm{APF}$, when the extrinsic stretching force starts to act on the wing, and observed at $24 \mathrm{~h} \mathrm{APF}$. Note that previous study has required two to three orders of magnitude higher concentrations of drugs in experiments using the pupal wing, likely reflecting the fact that the drug solution must pass through the cuticle (Tadashi Uemura, personal communication) ${ }^{46}$. Therefore, the concentration of Jasp in wing cells was $\sim 0.1-1 \mu \mathrm{M}$, which is within the range of values reported in other cells/tissues ${ }^{48,49}$
FRAP. sqhp-utrABD-GFP pupae at appropriate ages were mounted on an inverted confocal microscope (TCS SP8; Leica) equipped with a Leica HCX PL Apo ×63/ NA1.2 water-immersion objective. To photobleach utrABD-GFP along the AP junction, the region of the interest (ROI; $0.54 \mu \mathrm{m} \times 0.54 \mu \mathrm{m}$ ) was irradiated for $0.1 \mathrm{~s}$ using a two-photon laser tuned to an 880 -nm wavelength (Chameleon; Coherent). Images were collected at 0.437 -sec intervals. Fluorescence intensities (FIs) of ROIs were measured using ImageJ. FIs were normalized as the average FI at three subsequent time points immediately prior to photobleaching and subtracted the residual value immediately after photobleaching. A FRAP recovery curve was constructed by fitting the normalized FI to $\mathrm{FI}(t)=A\left(1-\exp \left(-\tau^{-1} t\right)\right)$ using MATLAB, from which the stable fraction of utrABD-GFP was calculated.

\section{Image analysis}

Subcellular distribution of proteins. To characterize directional bias in the subcellular distribution of AIP1-GFP, myo-II-GFP, and the AJ marker Da-catTagRFP, fluorescent signals along the AJ plane were extracted from a snapshot of live cells $(0.094 \mu \mathrm{m} /$ pixel). Subsequently, the following procedure was applied. First, the background signal was subtracted using the "subtract background" command $(r=100)$ in ImageJ. Second, to avoid counting the signals at the vertices for all associated junctions, we omitted the signals for 2 pixels at the ends of the junctions when calculating the mean signal intensity along each junction. Each junction was divided into twelve bins according to its angle relative to the $\mathrm{PD}$ axis, and the average signal intensity in each bin was plotted. In addition, we calculated the magnitude $R_{s}$ and the orientation $\Theta s$ of the polarity, defined as $R_{s}\langle s\rangle \mathrm{e}^{i 2 \Theta}=\left\langle\mathrm{se}^{\mathrm{i} 2 \theta}\right\rangle$ $-\langle s\rangle\left\langle\mathrm{e}^{i 2} \theta\right\rangle$ from a data set $\left\{s_{i j}, \theta_{i j}\right\}$, where $s_{i j}$ and $\theta_{i j}\left(0 \leq \theta_{i j}<\pi\right)$ are the fluorescent signal intensity and the angle of the contact surface between the $i$ th and $j$ th cells, respectively 7,47 . Larger values of $R_{s}$ indicate the stronger bias toward a particular axis.

When analyzing time-lapse images $(0.215 \mu \mathrm{m} / \mathrm{pixel})$, we set $r=20$ to subtract the background command. We omitted 1 pixel at the ends for junctions 6-8 pixels in length and 2 pixels at the ends for junctions longer than 8 pixels. The signal intensity along each junction was normalized to its temporal average.

In Fig. 31, Fig. 9a, and Supplementary Figure 2e, f, the animal was carrying one copy of AIP1-GFP, and the brightness-contrast was adjusted using different parameters compared to those used for other homozygous samples.

PCP polarity. We followed a protocol described in a previous study ${ }^{6}$ to measure the orientation of Fmi, Fmi-GFP, Dsh, and DE-cad polarities. Briefly, each pixel in an ROI was grouped into bins according to its angle relative to a cell center $\left(N_{\text {bin }}=36\right.$; the size of bin is $\left.\pi / N_{\text {bin }}\right)$, and the average signal intensity in each bin $\left\{s_{i j} \theta_{i}\right\}(j=1$, $2, \ldots, N_{\text {bin }}$ ) was measured. Subsequently, the mean polarity angle $\Theta s$ was calculated as $\cos 2 \Theta s=Q_{1} / \sqrt{Q_{1}^{2}+Q_{2}^{2}}$, where $Q_{1}=\sum_{i=1}^{N_{\text {bin }}} s_{i} \cos 2 \theta_{i}$ and $Q_{2}=\sum_{i=1}^{N_{\text {bin }}} s_{i} \sin 2 \theta_{i}$, respectively.

Hexagonal cell packing. DE-cad-GFP images were skeletonized using ImageJ. From the skeletonized images, the position and connectivity of vertices were extracted, and the polygonal distribution of cells was measured in OpenCV. If the distance between the centers of the end pixels of an edge was smaller than 3 pixels in length, the edge was regarded as a four-way vertex ${ }^{7}$.

Quantification of cell rearrangement process. Cell rearrangement was manually detected from time-lapse movies captured at 1-min intervals starting from $24 \mathrm{~h}$ $\mathrm{APF}$ at $25^{\circ} \mathrm{C}$ (WT, flr RNAi, DMSO, Japs) or at $20 \mathrm{~h} 30 \mathrm{~min}$ at $29^{\circ} \mathrm{C}$ (tsr RNAi). Subsequently, remodeling at each junction was automatically detected and manually assessed when necessary. By repeating this automatic detection and manual correction process, we estimated that $>90 \%$ of cell rearrangement in a filmed region were counted in this analysis. $\theta_{\text {final }}$ and $\theta_{\text {all }}$ represent the sets of angles from the final round and all rounds of cell rearrangement, respectively. The magnitudes $R_{\theta \text { final }}$ and $R_{\theta a l l}$ (one minus circular variance) and the mean orientation of $\theta$ are given as $R_{\theta} \mathrm{e}^{i 2 \Theta}=\left\langle\mathrm{e}^{i 2 \theta}\right\rangle\left(0 \leq \theta_{j}<\pi\right)$. Larger values of $R_{\theta}$ indicate the stronger directional bias. $N_{\text {seq }}$ and $N_{\text {all }}$ indicate the numbers of remodeled junctions and cell rearrangements in each movie, respectively. The frequency of cell rearrangement was calculated by dividing $N_{\text {seq }}$ and $N_{\text {all }}$ by $N_{\text {cell }}$, which is defined as the average value of the number of cells at the first and last time points of a movie.

Stress inference. Bayesian force/stress inference solves an inverse problem between forces (i.e., cell junction tension and cell pressure) and epithelial cell shape (i.e., position and connectivity of vertices that appear in an image $)^{47}$ and provides relative values for cell junction tensions and cell pressures, allowing the global stress tensor to be calculated by integrating tensions and pressures ${ }^{47,67}$. The accuracy and robustness of force/stress inference have been rigorously tested both in silico and in vivo ${ }^{47,68,69}$. In this study, we quantified the normal stress difference $\sigma_{\mathrm{A}} \equiv\left(\sigma_{x x}-\sigma_{y y}\right) / 2$ as a measure for the anisotropy of tissue stress from images of DE-cad-GFP.

Statistics. Data are presented as the mean \pm s.e.m. in plots of the signal intensity of AIP1-GFP, myo-II-GFP, and Da-cat-TagRFP in each angular bin and as the mean \pm s.d. in the other plots. $P$-values were calculated in R based on Welch's $t$-test 
(Figs. 1l, 2d, 4d, f, g, 9i, Supplementary Figure 5c, d, Supplementary Figure 8d, e), the Wilcoxon rank sum test (Fig. 9f), Dunnett's test (Fig. 2g, h, Supplementary Figure 4, Supplementary Figure 5a, b), the Steel test (Fig. 6j, Fig. 8e), the Steel-Dwass test (Figs. 7b, 8i, Supplementary Figure 10c, f), and a randomized version of Watson's test (Supplementary Figure 7c, e, g, i, Supplementary Figure 8b). Parametric tests were selected when the number of samples in each group was less than six and/or data follow the normal distribution. The Dunnett or Steel test was performed when analyzing multiple comparisons between the WT and other groups (the Steel test is a non-parametric equivalent of Dunnett's test). The Steel-Dwass test was performed when comparing every pair in all groups.

Data availability. The authors declare that the data supporting the findings of this study are available within the paper and its Supplementary files, and available from the corresponding author upon reasonable request. Plasmids and fly stocks generated in this study are available from the corresponding author.

Received: 25 October 2017 Accepted: 14 July 2018

Published online: 10 September 2018

\section{References}

1. Buchmann, A., Alber, M. \& Zartman, J. J. Sizing it up: the mechanical feedback hypothesis of organ growth regulation. Semin. Cell Dev. Biol. 35, 73-81 (2014)

2. Heisenberg, C. P. \& Bellaïche, Y. Forces in tissue morphogenesis and patterning. Cell 153, 948-962 (2013).

3. Shraiman, B. I. Mechanical feedback as a possible regulator of tissue growth. Proc. Natl Acad. Sci. USA 102, 3318-3323 (2005).

4. Le Goff, L., Rouault, H. \& Lecuit, T. A global pattern of mechanical stress polarizes cell divisions and cell shape in the growing Drosophila wing disc. Development 140, 4051-4059 (2013).

5. Pan, Y., Heemskerk, I., Ibar, C., Shraiman, B. I. \& Irvine, K. D. Differential growth triggers mechanical feedback that elevates Hippo signaling. Proc. Natl Acad. Sci. USA 113, E6974-E6983 (2016).

6. Aigouy, B. et al. Cell flow reorients the axis of planar polarity in the wing epithelium of Drosophila. Cell 142, 773-786 (2010).

7. Sugimura, K. \& Ishihara, S. The mechanical anisotropy in a tissue promotes ordering in hexagonal cell packing. Development 140, 4091-4101 (2013).

8. Etournay, R. et al. Interplay of cell dynamics and epithelial tension during morphogenesis of the Drosophila pupal wing. eLife 4, e07090 (2015).

9. Collinet, C., Rauzi, M., Lenne, P. F. \& Lecuit, T. Local and tissue-scale forces drive oriented junction growth during tissue extension. Nat. Cell Biol. 17, 1247-1258 (2015).

10. Aw, W. Y., Heck, B. W., Joyce, B. \& Devenport, D. Transient tissue-scale deformation coordinates alignment of planar cell polarity junctions in the mammalian skin. Curr. Biol. 26, 2090-2100 (2016)

11. Campinho, P. et al. Tension-oriented cell divisions limit anisotropic tissue tension in epithelial spreading during zebrafish epiboly. Nat. Cell Biol. 15, 1405-1414 (2013).

12. Choi, W. et al. Remodeling the zonula adherens in response to tension and the role of afadin in this response. J. Cell Biol. 213, 243-260 (2016).

13. West, J. J. et al. An actomyosin-Arf-GEF negative feedback loop for tissue elongation under stress. Curr. Biol. 27, 2260-2270 (2017).

14. Fletcher, D. A. \& Mullins, R. D. Cell mechanics and the cytoskeleton. Nature 463, 485-492 (2010)

15. Galkin, V. E., Orlova, A. \& Egelman, E. H. Actin filaments as tension sensors. Curr. Biol. 22, R96-R101 (2012).

16. Ehrlicher, A. J., Nakamura, F., Hartwig, J. H., Weitz, D. A. \& Stossel, T. P. Mechanical strain in actin networks regulates FilGAP and integrin binding to filamin A. Nature 478, 260-263 (2011)

17. Galkin, V. E. et al. Remodeling of actin filaments by ADF/cofilin proteins. Proc. Natl Acad. Sci. USA 108, 20568-20572 (2011).

18. Hayakawa, K., Tatsumi, H. \& Sokabe, M. Actin filaments function as a tension sensor by tension-dependent binding of cofilin to the filament. J. Cell Biol. 195, 721-727 (2011).

19. Ngo, K. X., Kodera, N., Katayama, E., Ando, T. \& Uyeda, T. Q. Cofilininduced unidirectional cooperative conformational changes in actin filaments revealed by high-speed atomic force microscopy. eLife 4, e04806 (2015).

20. Gardel, M. L. et al. Elastic behavior of cross-linked and bundled actin networks. Science 304, 1301-1305 (2004).

21. Chanet, S. et al. Actomyosin meshwork mechanosensing enables tissue shape to orient cell force. Nat. Commun. 8, 15014 (2017).

22. Rauzi, M., Lenne, P. F. \& Lecuit, T. Planar polarized actomyosin contractile flows control epithelial junction remodeling. Nature 468, 1110-1114 (2010).
23. Nishimura, T., Honda, H. \& Takeichi, M. Planar cell polarity links axes of spatial dynamics in neural-tube closure. Cell 149, 1084-1097 (2012).

24. Bardet, P. L. et al. PTEN controls junction lengthening and stability during cell rearrangement in epithelial tissue. Dev. Cell 25, 534-546 (2013).

25. Rozbicki, E. et al. Myosin-II-mediated cell shape changes and cell intercalation contribute to primitive streak formation. Nat. Cell Biol. 17, 397-408 (2015)

26. Matamoro-Vidal, A., Salazar-Ciudad, I. \& Houle, D. Making quantitative morphological variation from basic developmental processes: Where are we? The case of the Drosophila wing. Dev. Dyn. 244, 1058-1073 (2015).

27. Raufaste, C., Cox, S. J., Marmottant, P. \& Graner, F. Discrete rearranging disordered patterns: prediction of elastic and plastic behavior, and application to two-dimensional foams. Phys. Rev. E Stat. Nonlin Soft Matter Phys. 81 031404 (2010)

28. Guirao, B. \& Bellaiche, Y. Biomechanics of cell rearrangements in Drosophila Curr. Opin. Cell Biol. 48, 113-124 (2017).

29. Rodal, A. A., Tetreault, J. W., Lappalainen, P., Drubin, D. G. \& Amberg, D. C. Aiplp interacts with cofilin to disassemble actin filaments. J. Cell Biol. 145, 1251-1264 (1999)

30. Okada, K., Ravi, H., Smith, E. M. \& Goode, B. L. Aip1 and cofilin promote rapid turnover of yeast actin patches and cables: a coordinated mechanism for severing and capping filaments. Mol. Biol. Cell 17, 2855-2868 (2006).

31. Nadkarni, A. V. \& Brieher, W. M. Aip1 destabilizes cofilin-saturated actin filaments by severing and accelerating monomer dissociation from ends. Curr. Biol. 24, 2749-2757 (2014).

32. Jansen, S. et al. Single-molecule imaging of a three-component ordered actin disassembly mechanism. Nat. Commun. 6, 7202 (2015).

33. Okada, K., Obinata, T. \& Abe, H. XAIP1: a Xenopus homologue of yeast actin interacting protein 1 (AIP1), which induces disassembly of actin filaments cooperatively with ADF/cofilin family proteins. J. Cell Sci. 112, 1553-1565 (1999).

34. Ren, N., Charlton, J. \& Adler, P. N. The flare gene, which encodes the AIP1 protein of Drosophila, functions to regulate F-actin disassembly in pupal epidermal cells. Genetics 176, 2223-2234 (2007).

35. Lin, M. C., Galletta, B. J., Sept, D. \& Cooper, J. A. Overlapping and distinct functions for cofilin, coronin and Aip1 in actin dynamics in vivo. J. Cell Sci. 123, 1329-1342 (2010).

36. Chu, D. et al. AIP1 acts with cofilin to control actin dynamics during epithelial morphogenesis. Development 139, 3561-3571 (2012).

37. Luxenburg, C. et al. Wdr1-mediated cell shape dynamics and cortical tension are essential for epidermal planar cell polarity. Nat. Cell Biol. 17, 592-604 (2015).

38. Wu, J., Wang, H., Guo, X. \& Chen, J. Cofilin-mediated actin dynamics promotes actin bundle formation during Drosophila bristle development. $\mathrm{Mol}$. Biol. Cell 27, 2554-2564 (2016).

39. Buszczak, M. et al. The Carnegie protein trap library: a versatile tool for Drosophila developmental studies. Genetics 175, 1505-1531 (2007).

40. Morin, X., Daneman, R., Zavortink, M. \& China, W. A protein trap strategy to detect GFP-tagged proteins expressed from their endogenous loci in Drosophila. Proc. Natl Acad. Sci. USA 98, 15050-15055 (2001).

41. Krause, M., Dent, E. W., Bear, J. E., Loureiro, J. J. \& Gertler, F. B. Ena/VASP proteins: regulators of the actin cytoskeleton and cell migration. Annu Rev. Cell Dev. Biol. 19, 541-564 (2003).

42. Fernandez-Gonzalez, R., Simoes Sde, M., Röper, J. C., Eaton, S. \& Zallen, J. A. Myosin II dynamics are regulated by tension in intercalating cells. Dev. Cell 17, 736-743 (2009)

43. Hale, R., \& Strutt, D. Conservation of planar polarity pathway function across the animal kingdom. Annu Rev. Genet 49, 529-551 (2015).

44. Jenny, A., Reynolds-Kenneally, J., Das, G., Burnett, M. \& Mlodzik, M. Diego and Prickle regulate Frizzled planar cell polarity remodeling by competing for Dishevelled binding. Nat. Cell Biol. 7, 691-697 (2005).

45. Usui, T. et al. Flamingo, a seven-pass transmembrane cadherin, regulates planar cell polarity under the control of Frizzled. Cell 98, 585-595 (1999).

46. Shimada, Y., Yonemura, S., Ohkura, H., Strutt, D. \& Uemura, T. Polarized transport of Frizzled along the planar microtubule arrays in Drosophila wing epithelium. Dev. Cell 10, 209-222 (2006).

47. Ishihara, S. \& Sugimura, K. Bayesian inference of force dynamics during morphogenesis. J. Theor. Biol. 313, 201-211 (2012).

48. Bubb, M. R., Senderowicz, A. M., Sausville, E. A., Duncan, K. L. \& Korn, E. D. Jasplakinolide, a cytotoxic natural product, induces actin polymerization and competitively inhibits the binding of phalloidin to F-actin. J. Biol. Chem. 269, 14869-14871 (1994)

49. Tsuji, T., Miyoshi, T., Higashida, C., Narumiya, S. \& Watanabe, N. An order of magnitude faster AIP1-associated actin disruption than nucleation by the Arp2/3 complex in lamellipodia. PLoS ONE 4, e4921 (2009).

50. Classen, A. K., Anderson, K. I., Marois, E. \& Eaton, S. Hexagonal packing of Drosophila wing epithelial cells by the planar cell polarity pathway. Dev. Cell $\mathbf{9}$, 805-817 (2005). 
51. Axelrod, J. D. Unipolar membrane association of Dishevelled mediates Frizzled planar cell polarity signaling. Genes Dev. 15, 1182-1187 (2001).

52. Matsubara, D., Horiuchi, S. Y., Shimono, K., Usui, T. \& Uemura, T. The seven-pass transmembrane cadherin Flamingo controls dendritic selfavoidance via its binding to a LIM domain protein, Espinas, in Drosophila sensory neurons. Genes Dev. 25, 1982-1996 (2011).

53. Sawyer, J. K. et al. A contractile actomyosin network linked to adherens junctions by Canoe/afadin helps drive convergent extension. Mol. Biol. Cell 22, 2491-2508 (2011).

54. Sawyer, J. K., Harris, N. J., Slep, K. C., Gaul, U. \& Peifer, M. The Drosophila afadin homologue Canoe regulates linkage of the actin cytoskeleton to adherens junctions during apical constriction. J. Cell Biol. 186, 57-73 (2009).

55. Jodoin, J. N. et al. Stable force balance between epithelial cells arises from Factin turnover. Dev. Cell 35, 685-697 (2015).

56. Heer, N. C. \& Martin, A. C. Tension, contraction and tissue morphogenesis. Development 144, 4249-4260 (2017).

57. Noguchi, T. Q., Toya, R., Ueno, H., Tokuraku, K. \& Uyeda, T. Q. Screening of novel dominant negative mutant actins using glycine targeted scanning identifies G146V actin that cooperatively inhibits cofilin binding. Biochem Biophys. Res Commun. 396, 1006-1011 (2010).

58. Stachowiak, M. R. et al. Mechanism of cytokinetic contractile ring constriction in fission yeast. Dev. Cell 29, 547-561 (2014).

59. Lecuit, T. \& Yap, A. S. E-cadherin junctions as active mechanical integrators in tissue dynamics. Nat. Cell Biol. 17, 533-539 (2015).

60. Tee, Y. H. et al. Cellular chirality arising from the self-organization of the actin cytoskeleton. Nat. Cell Biol. 17, 445-457 (2015).

61. Huelsmann, S., Rintanen, N., Sethi, R., Brown, N. H. \& Ylanne, J. Evidence for the mechanosensor function of filamin in tissue development. Sci. Rep. 6 , 32798 (2016).

62. Bischof, J., Maeda, R. K., Hediger, M., Karch, F. \& Basler, K. An optimized transgenesis system for Drosophila using germ-line-specific phiC31 integrases. Proc. Natl Acad. Sci. USA 104, 3312-3317 (2007).

63. Takahashi, K., Matsuo, T., Katsube, T., Ueda, R. \& Yamamoto, D. Direct binding between two PDZ domain proteins Canoe and ZO-1 and their roles in regulation of the jun $\mathrm{N}$-terminal kinase pathway in Drosophila morphogenesis. Mech. Dev. 78, 97-111 (1998).

64. Parnas, D., Haghighi, A. P., Fetter, R. D., Kim, S. W. \& Goodman, C. S. Regulation of postsynaptic structure and protein localization by the Rho-type guanine nucleotide exchange factor dPix. Neuron 32, 415-424 (2001).

65. Shimada, Y., Usui, T., Yanagawa, S., Takeichi, M. \& Uemura, T. Asymmetric colocalization of Flamingo, a seven-pass transmembrane cadherin, and Dishevelled in planar cell polarization. Curr. Biol. 11, 859-863 (2001).

66. Guirao, B. et al. Unified quantitative characterization of epithelial tissue development. eLife 4, e08519 (2015).

67. Batchelor, G. K. The stress system in a suspension of force-free particles. J. Fluid Mech. 41, 545-570 (1970).

68. Ishihara, S. et al. Comparative study of non-invasive force and stress inference methods in tissue. Eur. Phys. J. E Soft Matter 36, 9859 (2013).

69. Sugimura, K., Bellaïche, Y., Graner, F., Marcq, P. \& Ishihara, S. Robustness of force and stress inference in an epithelial tissue. Conf. Proc. IEEE Eng. Med Biol. Soc. 2013, 2712-2715 (2013).

\section{Acknowledgements}

The authors would like to thank Yang Hong, Roger Kares, Thomas Lecuit, Tadashi Uemura, Daisuke Yamamoto, the Bloomington Stock Center, the Kyoto Stock Center, the Vienna Drosophila Resource Center, NIG-Fly, the Drosophila Genomics Resource Center, and the Developmental Studies Hybridoma Bank for reagents; Yuri Tsukahara, Miho Aruga, Kyoko Komano, Risa Matsui, and Machiko Tatsuno for technical assistance; and the iCeMS Imaging Center for imaging equipment. The authors would also like to thank the researchers listed in the Supplementary Methods for kindly providing the reagents used to screen candidate actin-binding proteins. Additionally, the authors thank Yohanns Bellaïche, Masahiro Sokabe, Kimihide Hayakawa, and Tetsuhisa Otani for intellectual input, Shuji Ishihara for technical advice regarding image analysis, and Yohanns Bellaïche, Masaki Arata, and James A. Hejna for critical reading of the manuscript. This study was financially supported by JSPS KAKENHI Grants (15H01321 and 17K15125), the JST PRESTO program (JPMJPR13A4), and the AMED PRIME program (JP17gm5810025).

\section{Author contributions}

K.I. and K.S. designed the research and analyzed the data. K.I. performed the experiments and K.S. assisted with the experiments. K.S. drafted the manuscript, with inputs from K.I. All authors approved the final manuscript.

\section{Additional information}

Supplementary Information accompanies this paper at https://doi.org/10.1038/s41467018-05605-7.

Competing interests: The authors declare no competing interests.

Reprints and permission information is available online at http://npg.nature.com/ reprintsandpermissions/

Publisher's note: Springer Nature remains neutral with regard to jurisdictional claims in published maps and institutional affiliations.

pen Access This article is licensed under a Creative Commons Attribution 4.0 International License, which permits use, sharing, adaptation, distribution and reproduction in any medium or format, as long as you give appropriate credit to the original author(s) and the source, provide a link to the Creative Commons license, and indicate if changes were made. The images or other third party material in this article are included in the article's Creative Commons license, unless indicated otherwise in a credit line to the material. If material is not included in the article's Creative Commons license and your intended use is not permitted by statutory regulation or exceeds the permitted use, you will need to obtain permission directly from the copyright holder. To view a copy of this license, visit http://creativecommons.org/ licenses/by/4.0/

(C) The Author(s) 2018 